\title{
Combined hybrid functional and DFT $+U$ calculations for metal chalcogenides
}

\author{
Mehmet Aras and Çetin Kilı, a) \\ Department of Physics, Gebze Institute of Technology, Gebze, Kocaeli 41400, \\ Turkey
}

(Dated: 6 July 2014)

\begin{abstract}
In the density-functional studies of materials with localized electronic states, the local/semilocal exchangecorrelation functionals are often either combined with a Hubbard parameter $U$ as in the LDA $+U$ method or mixed with a fraction of exactly computed (Fock) exchange energy yielding a hybrid functional. Although some inaccuracies of the semilocal density approximations are thus fixed to a certain extent, the improvements are not sufficient to make the predictions agree with the experimental data. Here we put forward the perspective that the hybrid functional scheme and the LDA $+U$ method should be treated as complementary, and propose to combine the range-separated (HSE) hybrid functional with the Hubbard $U$. We thus present a variety of $\mathrm{HSE}+U$ calculations for a set of II-VI semiconductors, consisting of zinc and cadmium monochalcogenides, along with comparison to the experimental data. Our findings imply that an optimal value $U^{*}$ of the Hubbard parameter could be determined, which ensures that the HSE $+U^{*}$ calculation reproduces the experimental band gap. It is shown that an improved description not only of the electronic structure but also of the crystal structure and energetics is obtained by adding the $U^{*}$ term to the HSE functional, proving the utility of $\mathrm{HSE}+U^{*}$ approach in modeling semiconductors with localized electronic states.
\end{abstract}

\section{INTRODUCTION}

Static correlation,, 12 arising from the tendency of electrons to distribute themselves over the various centers, is pronounced in materials containing localized $d$ or $f$ electrons such as some transition-metal or rare-earth compounds. The local density approximation ${ }^{3}(\mathrm{LDA})$ or the generalized gradient approximation ${ }^{4}$ (GGA) commonly employed in Kohn-Sham density functional theory ${ }^{3}$ (DFT) inherently assume a localized exchange-correlation hole, implying that static correlation is treated in an unrestrained manner in these approximations. ${ }^{5}$ Thus local or semilocal exchange-correlation energy $E_{\mathrm{xc}}$ functionals are often either combined with a Hubbard parameter $U$ as in the $\mathrm{LDA}+U$ method ${ }^{6}$ or mixed with a fraction $\alpha$ of exactly computed ${ }^{7}$ (Fock) exchange energy $E_{\mathrm{x}}^{\text {exact }}$, yielding a hybrid functional

$$
E_{\mathrm{xc}}^{\mathrm{hybrid}}=E_{\mathrm{x}}^{\text {exact }}+(1-\alpha)\left(E_{\mathrm{x}}^{\mathrm{GGA}}-E_{\mathrm{x}}^{\text {exact }}\right)+E_{\mathrm{c}}^{\mathrm{GGA}},
$$

where the second term models the static correlation energy ${ }^{[8]}$ For $\alpha>0$, the static correlation energy is reduced in favor of the suppression of electron fluctuations, leading to a better description for the localized electron states (as evidenced by the improved prediction of the binding energy of localized $d$ states, 19 band gaps,,$\frac{96}{19}$ and magnetic moments $\frac{12 \mid 19}{23}$ ). In the LDA $+U$ approach, where a $d$ ion is treated as an open system with fluctuation number of electrons,,$\sqrt[6]{ }$ a term including $U$ is added to the total energy, which penalizes more fluctuating configurations $\mathbf{2 4 | 2 5}$ and therefore leads to a better description of the localized states (as evidenced by the improved prediction of the binding energy of localized

\footnotetext{
a) E-mail: cetin_kilic@gyte.edu.tr
}

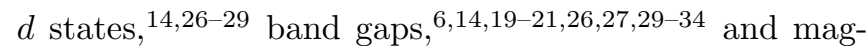

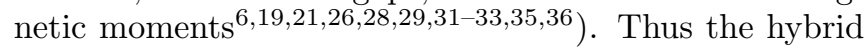
functional scheme and the LDA $+U$ approach could be regarded as alternative mean $\sqrt{37 / 38}$ for fixing inaccuracies of the semilocal density approximations, which result from insufficient localization of $d$ electrons. Indeed, it has recently been proposed $\frac{39 / 40}{4}$ to derive the value of $U$ from hybrid functional calculations. In contrast, we think it is appropriate to adopt a perspective where the hybridfunctional and $\mathrm{DFT}+U$ methods are treated complementary (inasmuch as they both reduce the static correlation energy), which led us to combine hybrid functionals with the Hubbard $U$. From a different point of view, this means that one of the two methods $(\mathrm{DFT}+U)$ is utilized to reduce the residual self-interaction error $\underline{41}$ of the other one (hybrid-functional), which is pragmatically justified. Furthermore, Ivády et al. (Ref. 42) have recently shown that a hybrid exchange-correlation potential could be cast into a mathematical form that is reminiscent of the on-site Hubbard potential for a subsystem of localized orbitals, providing theoretical justification for our methodology: An additional on-site $(\mathrm{DFT}+U)$ potential is added to the hybrid exchange-correlation potential, which is applied only to strictly localized states. This improves the physical description because localized $d$-band states and delocalized crystal states are differentiated in the hybrid-functional $+U$ approach, which are indifferent to the hybrid functional itself. It is also interesting in this regard to point out that the $\mathrm{DFT}+U$ and hybridfunctional methods could both be regarded as approximations to the $G W$ method,, 43 as articulated in Refs. 6 and 44, respectively. The incentive of using these two methods together is then to increase the level of approximation, provided that they are complementary.

It is usually necessary to perform a calibration $\sqrt{45 \mid 46}$ for the value of $U$ that is optimal with respect to the material properties under consideration. Besides $U$ is not only 

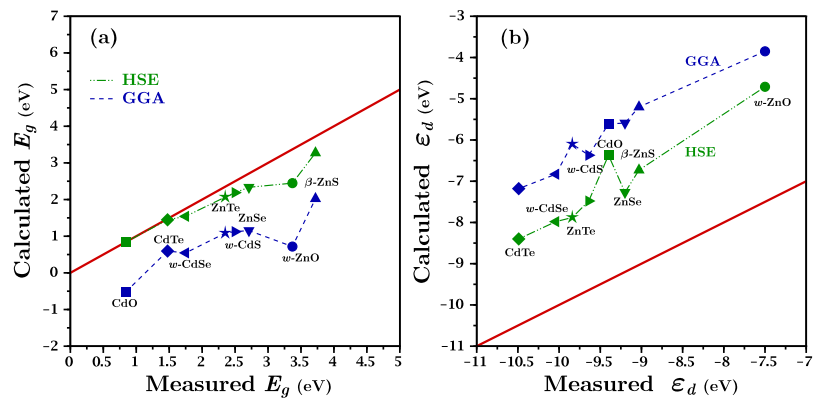

FIG. 1. (Color online) Calculated versus measured values of the band gap $E_{g}$ (a) and the $d$ band position $\varepsilon_{d}$ relative to the valence band maximum (b) for zinc and cadmium monochalcogenides. The experimental values of $E_{g}$ and $\varepsilon_{d}$ are taken from Refs. 60 62 and Refs. 63 and 64, respectively. The values obtained from the present GGA (HSE) calculations are connected by blue dashed (red dot-dashed) lines to guide the eye. The solid black lines passes through the experimental values.

element-specific ${ }^{45}$ but also material-specific. ${ }^{47 / 48}$ Thus it is appealing to employ a hybrid functional with an exchange mixing coefficient $\alpha$ that is in practice fixed to a single universal value, e.g., $\alpha=1 / 4$ in both globat 4 and range-separated Heyd-Scuseria-Ernzerhof ${ }^{50}$ (HSE) hybrid functionals. It should, however, be noted that setting the optimal value for $\alpha$ as $1 / 4$ in Ref. 49 was accomplished empirically (via error analysis of the atomization energies), which would not necessarily be optimal for other material properties ${ }^{51} \sqrt[54]{ }$ We found, in line with earlier reports $\frac{44 \mid 55156}{1}$ that the hybrid (HSE) functional calculations with $\alpha=1 / 4$ improve the prediction of both the $d$ band position $\varepsilon_{d}$ relative to the valence band maximum and the band gap $E_{g}$ but these improvements are not sufficient to make the predictions agree with the experimental data. This is demonstrated in Fig. 1 for zinc and cadmium monochalcogenides, where the calculated and measured values of $E_{g}$ (left panel) and $\varepsilon_{d}$ (right panel) are plotted with respect to each other. Figure 1(a) shows that (i) the improvement for the band gap is impressive for systems with a somewhat small band gap, and (ii) the band gap is still significantly underestimated for wide band gap semiconductors such as $\mathrm{ZnO}$. As explored in Appendix, both the GGA band gap error $\Delta E_{g}^{\mathrm{GGA}}$ and the HSE correction $E_{g}^{\mathrm{HSE}}-E_{g}^{\mathrm{GGA}}$ are inversely proportional to the high-frequency dielectric constant $\epsilon_{\infty}$ so that $\Delta E_{g}^{\mathrm{GGA}} \simeq A / \epsilon_{\infty}$ and $E_{g}^{\mathrm{HSE}}-E_{g}^{\mathrm{GGA}} \simeq A^{\prime} / \epsilon_{\infty}$, where the constants $A$ and $A^{\prime}$ satisfy $A^{\prime}<A$. Owing to the latter, the HSE improvement falls short for materials with relatively small dielectric constant (with the exception of $\mathrm{CdO}$ for which the HSE calculation yields the right direct and indirect band gaps, cf. Ref. 11). Figure 1(b) shows that the HSE-calculated $\varepsilon_{d}$ is still too high although there is a significant correction of about $1.3 \pm 0.4 \mathrm{eV}$. It should be noted that the prediction of $\varepsilon_{d}$ could further be improved by adding a Hubbard $U$ term to the hybrid functional, which would enable one to ad- just the $d$ band position. It is also interesting to note that the measured values of $\varepsilon_{d}$ could indeed be reproduced by using adjusted $U$ values, cf. Fig. 3 of Ref. 47, in the case of zinc monochalcogenides. These observations also motivate us to treat the hybrid functional scheme and the $\mathrm{DFT}+U$ method as complementary rather than alternative approaches. Accordingly, we propose here to combine the screened hybrid functional of Heyd, Scuseria, and Ernzerhof with the Hubbard $U$. The main advantage of the latter is that strictly localized and delocalized states are screened differently since only the former are subject to an additional on-site $(\mathrm{DFT}+U)$ potential. $\stackrel{42 \mid 57}{ }$ In contrast, localized and delocalized states are indifferent to the original HSE functional as long as the same set of parameters, viz. the exchange mixing coefficient $\alpha$ and the screening parameter $\omega$, are used for all states. Additionally, we regard $U$ as a semiempirical parameter, in line with the perspective $e^{58}$ that the Hubbard term added to the density functionals is essentially a phenomenological many-body correction. Our findings show that the $\mathrm{HSE}+U$ calculations performed by using an adjusted $U$ value reproduce the measured band gap and, at the same time, result in an improved physical description not only of the electronic structure but also of the crystal structure and energetics for the semiconductors with localized $d$ electrons. This is obviously very convenient for practical purposes such as setting the range of the electron chemical potential accurately in the point defect calculations, e.g., Ref. 59, It is also very convenient because it enables one to employ the measured $E_{g}$, instead of $\varepsilon_{d}$, in setting the $U$ value. Note that there is usually some scatter in the measured data for $\varepsilon_{d}$, which partly reflects the fact that the width of the $d$ bands is nonzero no matter how localized the states are.

The underestimation of the band gap in the HSE calculations, cf. Fig. 1(a), could partially be attributed to lacking the correlation part of the discontinuity of the exchange-correlation potential ${ }^{[65}$ Similarly, the discontinuity of the exchange-correlation potential is not fully restored in the LDA/GGA $+U$ calculations even though the $U$ term added to the density functionals yields a discontinuous contribution $[\sqrt[66]{ }$ It should also be commented that setting the right value of $U$ empirically is not straightforward because one needs to take accounts of hybridization and screening of $d$ electrons a priori. Furthermore, the measured value of $E_{g}$ could not be reproduced no matter how large a value of $U$ is used in the $\mathrm{LDA}+U$ calculations performed for zinc monochalcogenides, cf. Fig. 3 of Ref. 47. Our study provides a resolution to this difficulty with the aid of hybrid functional, and proves that an adequate $U$ value could be determined by simply matching the experimental band gap.

The rest of the paper is organized as follows: The next section is devoted to the method of calculation, which also summarizes the computational details. This is followed by a discussion of the calculation results before concluding remarks given in the last section. 


\section{METHOD}

All calculated properties reported here were obtained via semilocal or hybrid DFT calculations using the Perdew-Burke-Ernzerho" ${ }^{-1}$ (PBE) or Heyd-ScuseriaErnzerhof 50 (HSE) functionals, respectively. In the hybrid functional calculations, we employed the HSE0667 functional by setting the screening parameter $10 \mid 67 \omega=$ $0.207 \AA^{-1}$ (and exchange mixing coefficient $\alpha=0.25$ as implied in Section I). In the HSE $+U$ calculations we used the simplified (rotationally invariant) approach $\sqrt{30}$ where the difference between the on-site Coulomb $\bar{U}$ and exchange $\bar{J}$ parameters is employed as the effective Hubbard parameter $U=\bar{U}-\bar{J}$. We performed a variety of calculations for zinc and cadmium monochalcogenides by employing the projector augmented-wave (PAW) method, 68 as implemented in VASP code. 69170 The $2 \mathrm{~s}$ and $2 \mathrm{p}, 3 \mathrm{~s}$ and $3 \mathrm{p}, 4 \mathrm{~s}$ and $4 \mathrm{p}, 5 \mathrm{~s}$ and $5 \mathrm{p}, 3 \mathrm{~d}$ and $4 \mathrm{~s}$, and $4 \mathrm{~d}$ and $5 \mathrm{~s}$ states are treated as valence states for oxygen, sulfur, selenium, tellurium, zinc, and cadmium, respectively. Plane wave basis sets were used to represent the electronic states, which were determined by imposing a kinetic energy cutoff of $520 \mathrm{eV}$ for the systems that include oxygen atoms and $400 \mathrm{eV}$ for the rest of the systems.

We first carried out optimization of the crystal structures where concurrent relaxations of the cell volume and shape as well as the ionic positions were performed until the total energy was converged within $1 \mathrm{meV}$ and the maximum value of residual forces on atoms was reduced to be smaller than $0.01 \mathrm{eV} / \AA$. In these optimizations, we used the primitive unit cells of the crystals, whose Brillouin zones were sampled by $8 \times 8 \times 6$ (for the crystals with wurtzite structure) or $8 \times 8 \times 8$ or $9 \times 9 \times 9$ (for the crystals with rocksalt and zincblende structures) $\mathbf{k}$-point meshes generated according to Monkhorst-Pack scheme, ${ }^{71}$ enabling us to achieve convergence of the energy within $1 \mathrm{meV} /$ atom. Using the optimized crystal structures, we then performed band-structure and density-of-states calculations in order to obtain the band gap $E_{g}$ and the $d$ band position $\varepsilon_{d}$, respectively. Besides we performed geometry optimizations for the $\mathrm{O}_{2}$ and $\mathrm{S}_{8}$ molecules and the bulk solids of Se, Te, Zn, and Cd, and employed the respective equilibrium total energies in the computation of the formation energy $\Delta H_{f}$.

As indicated in Section II, we set the value of $U$ by reproducing the experimental value of the band gap in the HSE $+U$ calculations, which is justified in Section III. Thus, we carried out the $\mathrm{HSE}+U$ calculations for a range of $U$ values, and studied the calculated band gap as a function of $U$. Since our results showed that the variation of the band gap with $U$ is virtually linear, we performed a linear fit to obtain the value of $U$ that corresponds to the measured band gap. The value of $U$ obtained via this procedure, which is optimal in reproducing the experimental value of the band gap, is denoted by $U^{*}$. The HSE $+U$ calculation that yields the experimental, i.e., targeted, value of the band gap is named here as the
$\mathrm{HSE}+U^{*}$ calculation.

It should be mentioned that the HSE band energy differences depend on the value of the screening parameter $\omega$, which is not necessarily universal. It was, however, demonstrated ${ }^{72 \mid 73}$ that $\omega=0.207 \AA^{-1}$ as used in HSE06 is an average optimal value for which the band energy differences approximate rather accurately quasiparticle excitation energies, for a variety of semiconductors. Therefore, the HSE band energy differences are often $d i$ rectly compared to the experimental band gaps 44 (e.g., in order to demonstrate 15 the success of the HSE calculations in reproducing the experimental band gaps). In addition to this, as long as the HSE $+U$ approach could be regarded as an approximation to the $G W$ method, it would be preferential to use the quasiparticle energy differences (the $G W$-calculated band gaps) in our procedure for setting the value of $U^{*}$. However, the $G W$-calculated band gaps are usually in good agreement with the experimental band gaps (e.g., Ref. 74). It should, on the other hand, be also noted that the $G W @$ HSE calcula-
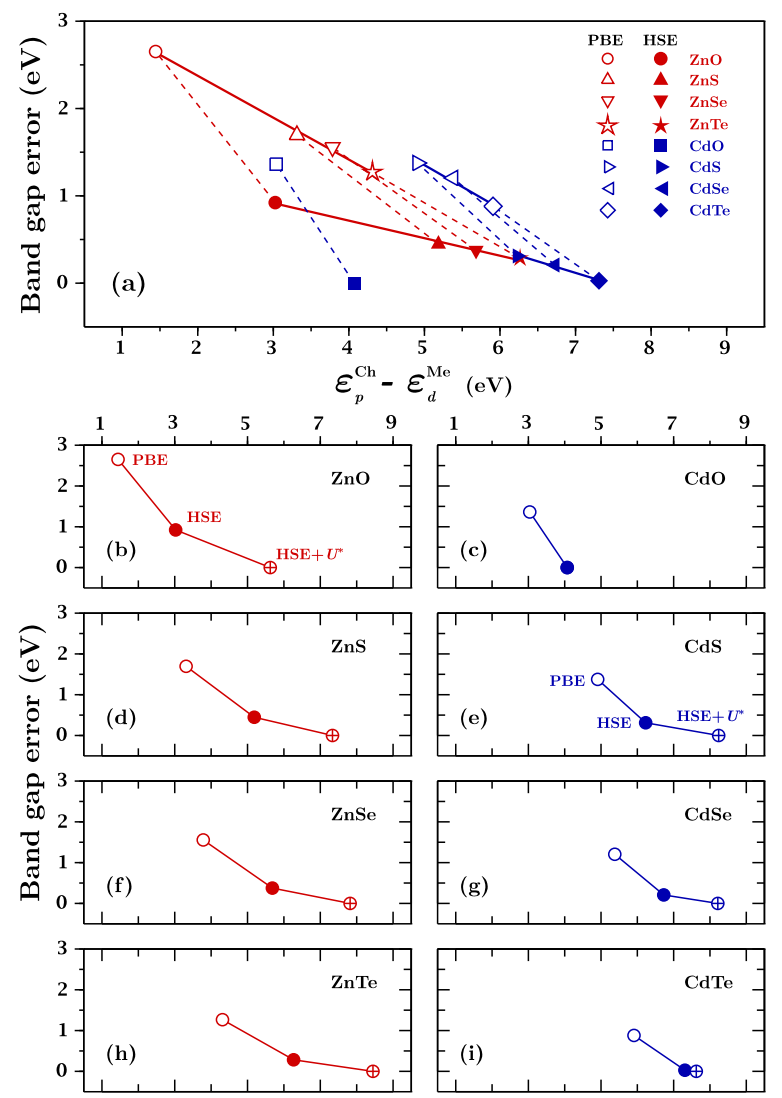

FIG. 2. (Color online) The band gap error $\Delta E_{g}$ versus the difference $\Delta \varepsilon_{p d}=\varepsilon_{p}^{\mathrm{Ch}}-\varepsilon_{d}^{\mathrm{Me}}$ for zinc and cadmium monochalcogenides. The PBE- and HSE-calculated values are marked by the empty and filled symbols, respectively, in the top-most panel (a). In the lower panels (b)-(i), the results of the combined HSE $+U^{*}(\oplus)$ calculations are presented together with those of the PBE (empty symbols) and HSE (filled symbols) calculations. 
tions overestimate the band gap of a number of semiconductors including CdS and ZnS (Ref. 56). Hence, we preferred to utilize the experimental band gaps instead of the $G W$-calculated energy differences, which is also convenient from a practical point of view since it enables one to avoid performing quasiparticle calculations that might easily become computationally exhaustive, especially for large-scale (e.g., defect) calculations.

\section{RESULTS AND DISCUSSION}

We first quantify the relationship between the band gap error $\Delta E_{g}$ in the GGA and HSE calculations and the position of $d$ level in the case of zinc and cadmium chalcogenides since the latter is, in effect, adjusted by varying the value of $U$. Figure 2(a) shows a plot of $\Delta E_{g}$ versus the difference $\Delta \varepsilon_{p d}=\varepsilon_{p}^{\mathrm{Ch}}-\varepsilon_{d}^{\mathrm{Me}}$, where $\varepsilon_{p}^{\mathrm{Ch}}$ and $\varepsilon_{d}^{\mathrm{Me}}$ denote the $p$ - and $d$-state energies of the chalcogen and metal atoms, respectively. In zinc and cadmium chalcogenides, the $d$ band is located below and next to the topmost valence band ${ }^{75}$ Thus, the valence-band maximum turns out to be above its actual position if the metal $d$ states are positioned too high (as in both the GGA and HSE calculations), which contributes to the underestimation of the band gap. The difference $\Delta \varepsilon_{p d}$ is therefore used here to quantify the relationship between the band gap error and the position of $d$ level. In Fig. 2(a), a linear trend is noticeable for each set of data, cf. the solid lines, with the exception of data points for $\mathrm{CdO}$. It is seen that the band gap error is proportional (with a negative slope) to $\Delta \varepsilon_{p d}$. We obtain, via fitting,

$$
\begin{aligned}
\Delta E_{g} & =-0.48 \Delta \varepsilon_{p d}+3.33 \quad(\mathrm{PBE}) \\
& =-0.20 \Delta \varepsilon_{p d}+1.52 \quad(\mathrm{HSE})
\end{aligned}
$$

for Zn chalcogenides, and

$$
\begin{array}{rlr}
\Delta E_{g} & =-0.50 \Delta \varepsilon_{p d}+3.84 \quad(\mathrm{PBE}) \\
& =-0.26 \Delta \varepsilon_{p d}+1.97 \quad(\mathrm{HSE})
\end{array}
$$

for $\mathrm{Cd}$ chalcogenides (excluding $\mathrm{CdO}$ ), where $\Delta E_{g}$ and $\Delta \varepsilon_{p d}$ are both in eV. It is clear, comparing the data points represented by empty (PBE) and filled (HSE) symbols connected by dashed lines, that the band gap error is reduced when the difference between the chalcogen $p$ - and metal $d$-state energies is increased. This applies to all II-VI semiconductors studied here, including $\mathrm{CdO}$. As shown in Figs. 2(b)-2(i), $\Delta \varepsilon_{p d}$ is significantly increased in the $\mathrm{HSE}+U^{*}$ calculations, making $\Delta E_{g}$ vanish. This is reassuring that the optimal Hubbard parameter $U^{*}$ could be determined by matching the experimental band gap.

We now determine the $U^{*}$ values that corresponds to vanishing $\Delta E_{g}$ for the II-VI semiconductors under consideration. Thus, the results of $\mathrm{HSE}+U$ calculations for a range of $U$ values are given in Fig. 3 where the calculated band gap is plotted as a function of $U$. Note that the variation of the band gap with the effective Hubbard parameter is virtually linear (with a different slope for

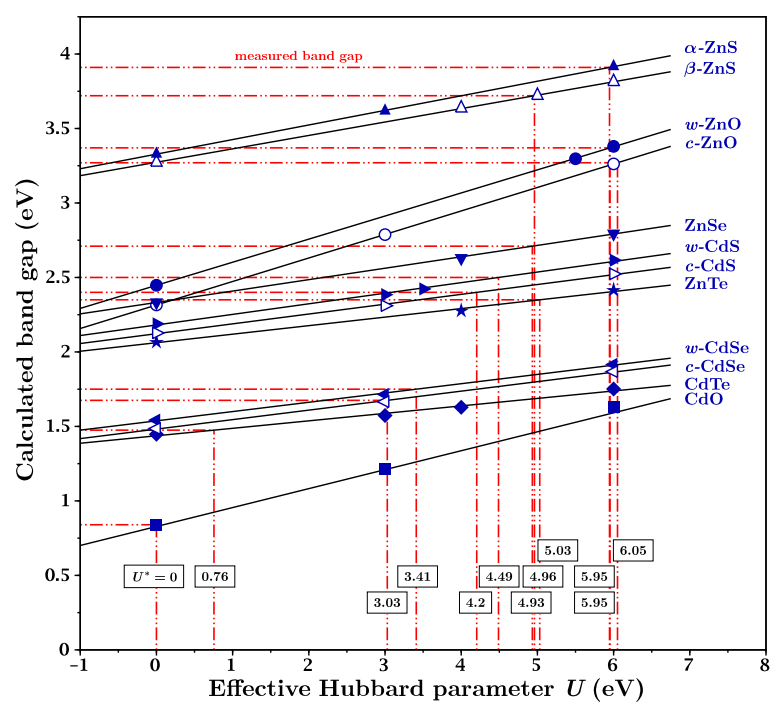

FIG. 3. (Color online) The band gaps $E_{g}$ obtained in our $\mathrm{HSE}+U$ calculations as a function of the effective Hubbard parameter $U$ for zinc and cadmium monochalcogenides. The symbols represent the calculated $E_{g}$ values, and the solid lines connecting the symbols represent linear fits to the calculated points. The vertical dot-dashed lines mark the values for the optimal Hubbard parameter $U^{*}$ in $\mathrm{eV}$, which correspond to the experimental $E_{g}$ values (marked by the horizontal dotdashed lines).

TABLE I. The optimal Hubbard parameter $U^{*}$, the experimental band gap $E_{g}$, and the HSE band gap error $\Delta E_{g}^{\mathrm{HSE}}$ (all in $\mathrm{eV}$ ) for zinc and cadmium monochalcogenides.

\begin{tabular}{llccc}
\hline \hline Semiconductor & Crystal structure & $U^{*}$ & $E_{g}$ & $\Delta E_{g}^{\mathrm{HSE}}$ \\
\hline $\mathrm{CdO}$ & rocksalt & 0.0 & 0.84 & 0.00 \\
$\mathrm{CdTe}$ & zincblende & 0.8 & 1.48 & 0.03 \\
$c$-CdSe & zincblende & 3.0 & 1.68 & 0.19 \\
$w$-CdSe & wurtzite & 3.4 & 1.75 & 0.21 \\
$\mathrm{ZnTe}$ & zincblende & 5.0 & 2.35 & 0.28 \\
$c$-CdS & zincblende & 4.2 & 2.40 & 0.27 \\
$w$-CdS & wurtzite & 4.5 & 2.50 & 0.31 \\
$\mathrm{ZnSe}$ & zincblende & 5.0 & 2.71 & 0.38 \\
$c$-ZnO & zincblende & 6.1 & 3.27 & 0.95 \\
$w$-ZnO & wurtzite & 6.0 & 3.37 & 0.92 \\
$\beta$-ZnS & zincblende & 5.0 & 3.72 & 0.45 \\
$\alpha$-ZnS & wurtzite & 6.0 & 3.91 & 0.58 \\
\hline \hline
\end{tabular}

each system). For each compound, a linear fit is thus performed, which yields the solid lines in Fig. 3 . The $U^{*}$ values are marked by vertical dot-dashed lines, which correspond to the measured band gap (marked by horizontal dot-dashed lines). Table I gives the optimal Hubbard parameters and corresponding band gaps for zinc and cadmium monochalcogenides. It should be remarked that one obtains $U^{*}=0$ for $\mathrm{CdO}$ since the measured value of the band gap of $\mathrm{CdO}$ is reproduced already in the HSE calculation, as mentioned in Section 1 .

Next we compare the values of $\Delta E_{g}$ and $\Delta \varepsilon_{p d}$ obtained in the $\mathrm{HSE}+U^{*}$ calculations to those obtained in the PBE 


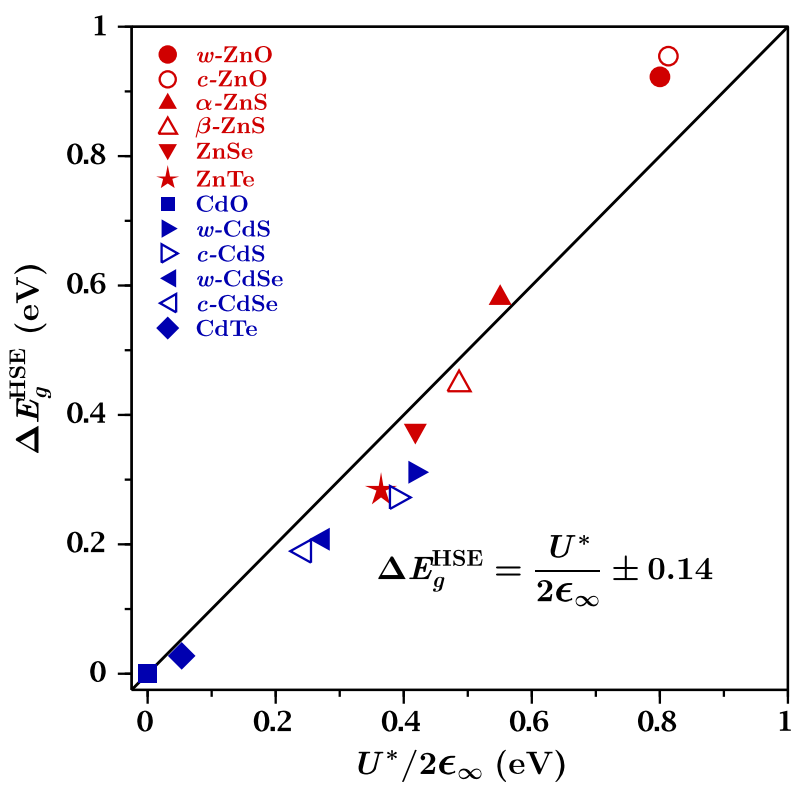

FIG. 4. (Color online) The HSE band gap error $\Delta E_{g}^{\mathrm{HSE}}$ versus the ratio $U^{*} / 2 \epsilon_{\infty}$.

and HSE calculations. Figures 2(b)-2(i) show a plot of the band gap error $\Delta E_{g}$ versus the difference $\Delta \varepsilon_{p d}$ for the II-VI semiconductors under consideration. As already noted, the HSE calculations yield an increased value for $\Delta \varepsilon_{p d}$ in association with a reduced band gap error, in comparison to the PBE calculations. The difference $\Delta \varepsilon_{p d}$ is further increased in the HSE $+U$ calculations, reducing the band gap error further. Having $U=U^{*}$ in this trend makes $\Delta E_{g}$ vanish, with adequate increase of $\Delta \varepsilon_{p d}$.

It is seen in Table I that the larger $E_{g}$ the greater $U^{*}$ (with few exceptions). This implies that employing a large (small) $U^{*}$ would be necessary for a wide (narrow) band gap semiconductor for which the HSE band gap error $\Delta E_{g}^{\mathrm{HSE}}$ is rather large (small), cf. Figure 1(a). Thus, having a large band gap error in the HSE calculation necessitates using a large $U^{*}$ for correction. Furthermore, there appears to be a roughly monotonic relationship between $U^{*}$ and $\Delta E_{g}^{\mathrm{HSE}}$, cf. Table $\mathrm{I}$. Our analysis presented in Fig. 4 shows that this relationship could be quantified by taking into account the screening effects through the high-frequency dielectric constant $\epsilon_{\infty}$. A plot of $\Delta E_{g}^{\mathrm{HSE}}$ versus $U^{*} / 2 \epsilon_{\infty}$ is given in Fig. 4 where all data points satisfy

$$
\Delta E_{g}^{\mathrm{HSE}}=\frac{U^{*}}{2 \epsilon_{\infty}} \pm 0.14 \mathrm{eV} .
$$

Here both $U^{*}$ and $\Delta E_{g}^{\mathrm{HSE}}$ are in $\mathrm{eV}$. Note that the shift in the occupied (unoccupied) $d$ state energies due to the $U^{*}$ term would be $-U^{*} / 2\left(U^{*} / 2\right)$ if the hybridization and screening effects are ignored 66 Thus, the correction to the band gap would be proportional to $U^{*} / 2$, ignoring the dielectric screening, for the II-VI semiconductors studied here since their lower conduction bands have virtually no contribution from the metal $d$ states. ${ }^{75}$ On the other hand, the band gap correction needs to be scaled by $\epsilon_{\infty}$ in order to reflect the dielectric screening of the Coulomb potential in a solid ${ }^{76}$ Thus, the $U^{*}$ term added to the hybrid (HSE) functional results in a correction of $U^{*} / 2 \epsilon_{\infty}$ to the band gap. This explanation justifies our means of setting the value of $U^{*}$ by matching the experimental band gap. It also implies that an approximate value for the optimal Hubbard parameter could a priori be obtained by inverting Eq. (4), i.e., $U^{*} \approx 2 \epsilon_{\infty}\left(E_{g}-E_{g}^{\mathrm{HSE}}\right)$, provided that the experimental and HSE-calculated band gaps $E_{g}$ and $E_{g}^{\mathrm{HSE}}$ as well as the high-frequency dielectric constant $\epsilon_{\infty}$ are available. Note that the hybridfunctional calculations could be utilized to obtain $\epsilon_{\infty}$ when the experimental data is not available, cf. Table I of Ref. 77 .

It is interesting to point out that one could assign a single $U^{*}$ value of $\sim 5 \mathrm{eV}$ for $\mathrm{ZnTe}, \mathrm{ZnSe}$ and $\beta$ - ZnS while $U^{*} \sim 6 \mathrm{eV}$ for $c-\mathrm{ZnO}, w-\mathrm{ZnO}$ and $\alpha-\mathrm{ZnS}$, cf. Table I. Thus, a mean value of $U_{\mathrm{Zn}}^{*} \approx 5.5 \mathrm{eV}$ appears to be adequate for all $\mathrm{Zn}$ compounds studied here. It is clearly pleasing to obtain a single (universal) $U^{*}$ value for $\mathrm{Zn}$, which is almost independent of the composition or crystal structure of the relevant zinc compounds, for its practical importance since it would allow one to set $U_{\mathrm{Zn}}^{*} \approx 5.5 \mathrm{eV}$ in the studies on alloyed systems made of $\mathrm{Zn}, \mathrm{O}, \mathrm{S}, \mathrm{Se}, \mathrm{Te}$ atoms.

In order to assess the improvement of the $\mathrm{HSE}+U$ approach in relation to the general physical description of the foregoing semiconductors, we computed the mean error in (i) the optimized crystal structures, (ii) the $d$ band positions, and (iii) the formation energies of the metal chalcogenides under consideration. Accordingly, a comparison of errors in the predictions of the HSE $+U^{*}$, HSE and PBE calculations is presented Fig. 5 where the comparison is performed for the unit cell volume $V$ [in Fig. 5(a)], for the ratio $c / a$ of (wurtzite) lattice parameters $a$ and $c$ [in Fig. 5(b)], for the internal parameter $u$ of wurtzite structure [in Fig. 5(c)], for the $d$ band position $\varepsilon_{d}$ [in Fig. 5(d)], and for the formation energy $\Delta H_{f}$ [in Fig. 5(e)]. Our analysis reveals the following:

First, we see in Figs. 5(a)-(c) that the optimization of the crystal structure via HSE or $\mathrm{HSE}+U^{*}$ calculation results in a similarly more accurate description, in comparison to the PBE calculations. Thus, the HSE $+U^{*}$ calculations seem to preserve the accuracy of the HSE calculations in the crystal structure optimizations.

Secondly, Fig. 5(d) shows that there is a significant correction to the $d$ band position thanks to adding $U^{*}$ term to the HSE functional: The mean error in the $\varepsilon_{d}$ prediction becomes $\sim 0.6 \mathrm{eV}$ in the $\mathrm{HSE}+U^{*}$ calculations, compared to $\sim 2.3(3.6) \mathrm{eV}$ in the HSE (PBE) calculations. It should also be noted that the variation of the difference $\Delta \varepsilon_{d}^{*}=\varepsilon_{d}^{\mathrm{HSE}+U^{*}}-\varepsilon_{d}^{\mathrm{HSE}}$ with $U^{*}$ is almost linear $\frac{78}{78}$ wich is consistent with $\Delta \varepsilon_{d}^{*} \approx-0.35 U^{*}$, where both $\Delta \varepsilon_{d}^{*}$ and $U^{*}$ are in $\mathrm{eV}$. Thus, using a larger $U^{*}$ yields a larger correction to $\varepsilon_{d}$, shifting the $d$ band to 

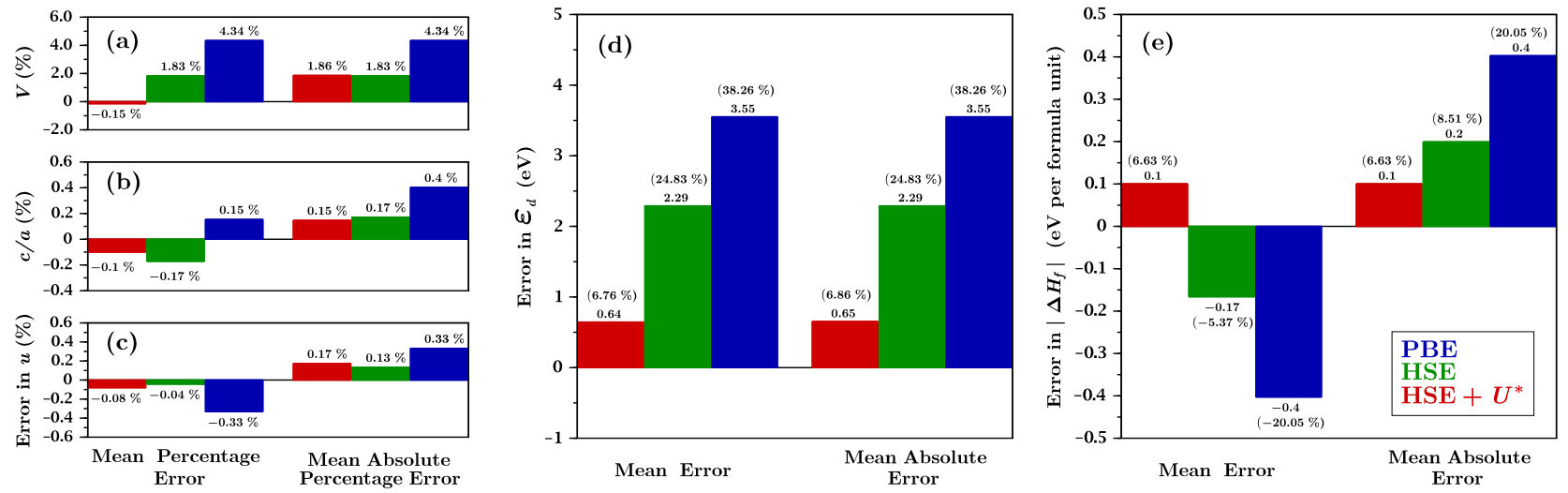

FIG. 5. (Color online) A comparison of errors in the predictions via HSE $+U^{*}$ (red bars), HSE (green bars) and PBE (blue bars) calculations for the unit cell volume $V$ (a), the ratio $c / a$ of lattice parameters $a$ and $c$ (b), the internal lattice parameter $u(\mathrm{c})$, the $d$ band position $\varepsilon_{d}(\mathrm{~d})$, and the absolute value of formation energy $\left|\Delta H_{f}\right|(\mathrm{e})$.

a lower position that is closer to its experimental location. Recall that employing a larger $U^{*}$ is necessary for the systems with a larger HSE band gap error (cf. Table I). Hence, the improvement in predicting the $d$ band position via $\mathrm{HSE}+U^{*}$ calculations is warranted since the value of $U^{*}$ is determined by matching the experimental band gap.

Finally, as for the improvement of the HSE $+U$ approach in the prediction of formation energies, Fig. 5(e) shows that the mean absolute error in $\left|\Delta H_{f}\right|$ is on the order of $\sim 0.1,0.2$, and $0.4 \mathrm{eV}$ per formula unit in the $\mathrm{HSE}+U^{*}, \mathrm{HSE}$, and PBE calculations, respectively. Thus, the $\mathrm{HSE}+U^{*}$ calculations result in a more accurate description of crystal energetics of zinc and cadmium monochalcogenides, compared to the HSE and PBE calculations. Note that the mean error in $\left|\Delta H_{f}\right|$ turns out to be positive in the $\mathrm{HSE}+U^{*}$ calculations, which is negative in the HSE calculations. This indicates that the error in the formation energies could be further reduced, whenever necessary, by re-adjusting the value of $U^{*}$.

\section{CONCLUSION}

In this work, we treated the hybrid functional scheme and the $\mathrm{DFT}+U$ method as complementary rather than alternative approaches in studying a set of II-VI semiconductors with localized $d$ states. This led us to introduce the $\mathrm{HSE}+U$ approach where the range-separated HSE hybrid functional is combined with the Hubbard $U$. Furthermore, we regarded $U$ as a semiempirical parameter. This enabled us to determine an optimal value $U^{*}$ of the Hubbard parameter, for which the $\mathrm{HSE}+U$ calculation yields a targeted (e.g., experimental) value of the band gap. We find that the correction to the band gap due to the additional $U^{*}$ term is roughly given by $U^{*} / 2 \epsilon_{\infty}$, which is in line with theoretical reasoning. The results of a variety of $\mathrm{HSE}+U^{*}$ calculations performed for zinc and cadmium monochalcogenides, viz., a subset of the semiconductors with localized $d$ states, indicate that an improved description of the electronic structure as well as crystal structure and energetics is obtained in these calculations, compared to the hybrid functional calculations employing the HSE functional without an additional Hubbard term. The present study thus shows that adding the $U^{*}$ term to the HSE functional leads to more accurate prediction of both the electronic and crystal structures of II-VI semiconductors with localized states.

\section{ACKNOWLEDGMENTS}

The numerical calculations reported here were carried out at the High Performance and Grid Computing Center (TRUBA Resources) of TUBITAK ULAKBIM.

\section{Appendix: Band gap error in DFT and hybrid-functional calculations}

Figure 6(a) shows a plot of the band gap error $\Delta E_{g}^{\mathrm{PBE}}=E_{g}-E_{g}^{\mathrm{PBE}}$ in the GGA calculation versus the inverse high-frequency dielectric constant $1 / \epsilon_{\infty}$, where a nearly linear trend is noticeable for each set of data. It is clear that the band gap error is larger for materials with smaller $\epsilon_{\infty}$. This is in line with the finding 8182 that the self-energy correction to the DFT-calculated band gap is inversely proportional to the high-frequency dielectric constant. Employing the LDA-calculated band gaps, it was found ${ }^{81}$ that the product $\epsilon_{\infty} \Delta E_{g}^{\mathrm{LDA}} \approx 9.1 \pm 0.2 \mathrm{eV}$ is a material-independent constant. We find that the product $\epsilon_{\infty} \Delta E_{g}^{\mathrm{PBE}}=A$ is also roughly a constant, but with a different value for each class of systems: $A_{\mathrm{Zn}}=$ $9.4 \pm 0.6 \mathrm{eV}$ and $A_{\mathrm{Cd}}=7.2 \pm 0.5 \mathrm{eV}$ for $\mathrm{Zn}$ and Cd chalcogenides, respectively. Note that the data points in red 


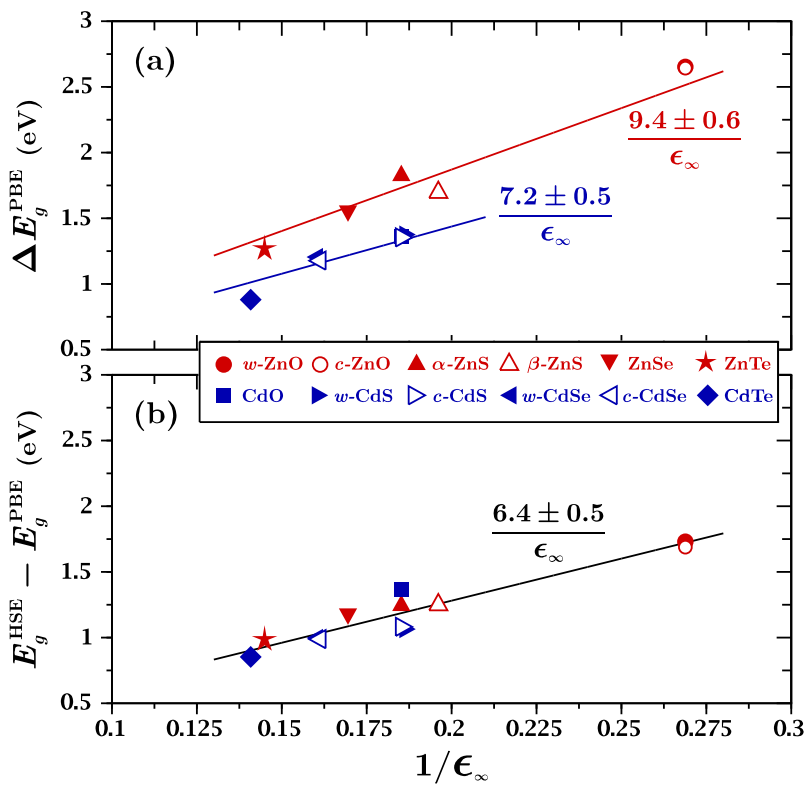

FIG. 6. (Color online) The band gap error $\Delta E_{g}^{\mathrm{PBE}}$ in the PBE calculations (a) and the difference $E_{g}^{\mathrm{HSE}}-E_{g}^{\mathrm{PBE}} \mathrm{HSE}-$ and PBE-calculated band gaps (b) versus the inverse dielectric constant $1 / \epsilon_{\infty}$. The experimental values of $E_{g}$ and $\epsilon_{\infty}$ are taken from Refs. 60,62 and Refs. 79 and 80 , respectively.

(blue) in Fig. 6(a) are consistent with the red (blue) line given by $\Delta E_{g}^{\mathrm{PBE}}=A_{\mathrm{Zn}} / \epsilon_{\infty}\left(\Delta E_{g}^{\mathrm{PBE}}=A_{\mathrm{Cd}} / \epsilon_{\infty}\right)$. On the other hand, our results presented in Fig. 6(b) show that the difference $E_{g}^{\mathrm{HSE}}-E_{g}^{\mathrm{PBE}}$ is also inversely proportional to $\epsilon_{\infty}$ so that $E_{q}^{\mathrm{HSE}}-E_{g}^{\mathrm{PBE}} \approx A^{\prime} / \epsilon_{\infty}$, where $A^{\prime}=6.4 \pm 0.5 \mathrm{eV}$ is a material-independent constant. Combining $\Delta E_{g}^{\mathrm{PBE}}-\Delta E_{g}^{\mathrm{HSE}}=E_{g}^{\mathrm{HSE}}-E_{g}^{\mathrm{PBE}} \approx A^{\prime} / \epsilon_{\infty}$ [cf. Fig. 6(b)] and $\Delta E_{g}^{\mathrm{PBE}} \approx A / \epsilon_{\infty}$ [cf. Fig. 6(a)], we obtain $\Delta E_{g}^{\mathrm{HSE}} \approx\left(A-A^{\prime}\right) / \epsilon_{\infty}$. Note that both $A_{\mathrm{Zn}}$ and $A_{\mathrm{Cd}}$ are greater than $A^{\prime}$. Thus, the band gap is underestimated in the HSE calculations in proportionality with $1 / \epsilon_{\infty}$ albeit there is a significant improvement in comparison to the respective GGA calculations.

${ }^{1}$ N. C. Handy and A. J. Cohen, Mol. Phys. 99, 403-412 (2001).

${ }^{2}$ A. D. Becke, J. Chem. Phys. 138, 074109 (2013)

${ }^{3}$ W. Kohn and L. J. Sham, Phys. Rev. 140, A1133-A1138 (1965)

${ }^{4}$ J. P. Perdew, K. Burke, and M. Ernzerhof, Phys. Rev. Lett. 77, 3865-3868 (1996)

${ }^{5}$ A. D. Becke, J. Chem. Phys. 122, 064101 (2005)

${ }^{6}$ V. I. Anisimov, F. Aryasetiawan, and A. I. Lichtenstein, J. Phys.: Condens. Matter 9, 767 (1997).

${ }^{7}$ A. D. Becke, J. Chem. Phys. 98, 1372-1377 (1993)

${ }^{8}$ G. I. Csonka, J. P. Perdew, and A. Ruzsinszky, J. Chem. Theory Comput. 6, 3688-3703 (2010)

${ }^{9}$ F. Oba, A. Togo, I. Tanaka, J. Paier, and G. Kresse, Phys. Rev. B 77, $245202(2008)$

${ }^{10}$ J. Wróbel, K. J. Kurzydłowski, K. Hummer, G. Kresse, and J. Piechota, Phys. Rev. B 80, 155124 (2009).

${ }^{11}$ M. Burbano, D. O. Scanlon, and G. W. Watson, J. Am. Chem. Soc. 133, 15065-15072 (2011)

${ }^{12}$ Z. D. Pozun and G. Henkelman, J. Chem. Phys. 134, 224706 (2011)
${ }^{13} \mathrm{~J}$. Li, J. Gayles, N. Kioussis, Z. Zhang, C. Grein, and F. Aqariden, J. Electron. Mater. 41, 2745-2753 (2012).

${ }^{14}$ Y. Zhang, X. Yuan, X. Sun, and W. Zhang, Solid State Commun. 152, $588-592(2012)$

${ }^{15}$ J. Heyd, J. E. Peralta, G. E. Scuseria, and R. L. Martin, J. Chem. Phys. 123, 174101 (2005).

${ }^{10}$ M. Marsman, J. Paier, A. Stroppa, and G. Kresse, J. Phys.: Condens. Matter 20, 064201 (2008)

${ }^{17}$ J.-C. Wu, J. Zheng, C. L. Zacherl, P. Wu, Z.-K. Liu, and R. Xu, J. Phys. Chem. C 115, 19741-19748 (2011)

${ }^{18}$ M. J. Lucero, T. M. Henderson, and G. E. Scuseria, J. Phys.: Condens. Matter 24, 145504 (2012)

${ }^{19}$ D. K. Kanan and E. A. Carter, J. Phys. Chem. C 116, 9876-9887 (2012)

${ }^{20} \mathrm{C}$. Rödl, F. Fuchs, J. Furthmüller, and F. Bechstedt, Phys. Rev. B 79, 235114 (2009)

${ }^{21}$ P. Liao and E. A. Carter, Phys. Chem. Chem. Phys. 13, 1518915199 (2011)

${ }^{22}$ H. Chen and J. H. Harding, Phys. Rev. B 85, 115127 (2012)

${ }^{23}$ M. Heinemann, B. Eifert, and C. Heiliger, Phys. Rev. B 87, 115111 (2013)

${ }^{24}$ J. P. Perdew, A. Ruzsinszky, G. I. Csonka, O. A. Vydrov, G. E. Scuseria, V. N. Staroverov, and J. Tao, Phys. Rev. A 76, 040501 (2007)

${ }^{25}$ J. P. Perdew, A. Ruzsinszky, L. A. Constantin, J. Sun, and G. I. Csonka, J. Chem. Theory Comput. 5, 902-908 (2009)

${ }^{26}$ A. Rohrbach, J. Hafner, and G. Kresse, J. Phys.: Condens. Matter 15, 979 (2003)

${ }^{27}$ T. Miyake, P. Zhang, M. L. Cohen, and S. G. Louie, Phys. Rev. B 74, 245213 (2006)

${ }^{28}$ M. Petersen, J. Hafner, and M. Marsman, J. Phys.: Condens. Matter 18, $7021(2006)$

${ }^{29}$ W.-B. Zhang, J. Li, and B.-Y. Tang, J. Chem. Phys. 138, 244703 (2013)

${ }^{30}$ S. L. Dudarev, G. A. Botton, S. Y. Savrasov, C. J. Humphreys, and A. P. Sutton, Phys. Rev. B 57, 1505-1509 (1998)

${ }^{31}$ A. Rohrbach, J. Hafner, and G. Kresse, Phys. Rev. B 70, 125426 (2004)

${ }^{32}$ F. Gupta, G. Brillant, and A. Pasturel, Philos. Mag. 87, 2561$2569(2007)$

${ }^{33}$ A. J. Devey, R. Grau-Crespo, and N. H. de Leeuw, Phys. Rev. B 79, 195126 (2009)

${ }^{34}$ M. E. Arroyo-de Dompablo, A. Morales-Garca, and M. Taravillo, J. Chem. Phys. 135, 054503 (2011)

${ }^{35}$ G. Rollmann, A. Rohrbach, P. Entel, and J. Hafner, Phys. Rev. B 69, 165107 (2004)

${ }^{36}$ L. Wang, T. Maxisch, and G. Ceder, Phys. Rev. B 73, 195107 (2006)

${ }^{37}$ F. Tran, P. Blaha, K. Schwarz, and P. Novák, Phys. Rev. B 74, $155108(2006)$

${ }^{38}$ F. Jollet, G. Jomard, B. Amadon, J. P. Crocombette, and D. Torumba, Phys. Rev. B 80, 235109 (2009)

${ }^{39}$ J. Hong, A. Stroppa, J. Íñiguez, S. Picozzi, and D. Vanderbilt, Phys. Rev. B 85, 054417 (2012)

${ }^{40}$ A. N. Andriotis, G. Mpourmpakis, S. Lisenkov, R. M. Sheetz, and M. Menon, Phys. Status Solidi B 250, 356-363 (2013)

${ }^{41}$ J. P. Perdew and A. Zunger, Phys. Rev. B 23, 5048-5079 (1981)

${ }^{42}$ V. Ivády, R. Armiento, K. Szász, E. Janzén, A. Gali, and I. A. Abrikosov, ArXiv e-prints (2014), arXiv:1406.2944 [condmat.str-el]

${ }^{43}$ L. Hedin, Phys. Rev. 139, A796-A823 (1965)

${ }^{44}$ T. M. Henderson, J. Paier, and G. E. Scuseria, Phys. Status Solidi B 248, 767-774 (2011)

${ }^{45}$ S. Lutfalla, V. Shapovalov, and A. T. Bell, J. Chem. Theory and Comput. 7, 2218-2223 (2011)

${ }^{46}$ M. D. Krcha and M. J. Janik, Int. J. Quantum Chem. 114, 8-13 (2014)

${ }^{4 r}$ S. Z. Karazhanov, P. Ravindran, A. Kjekshus, H. Fjellvåg, U. Grossner, and B. G. Svensson, J. Appl. Phys. 100, 043709 (2006) 
${ }^{48}$ G. Barcaro, I. O. Thomas, and A. Fortunelli, J. Chem. Phys. 132, 124703 (2010).

${ }^{49}$ J. P. Perdew, M. Ernzerhof, and K. Burke, J. Chem. Phys. 105, 9982-9985 (1996)

${ }^{0}$ J. Heyd, G. E. Scuseria, and M. Ernzerhof, J. Chem. Phys. 118, 8207-8215 (2003).

${ }^{51}$ O. A. Vydrov, J. Heyd, A. V. Krukau, and G. E. Scuseria, J. Chem. Phys. 125, 074106 (2006)

${ }^{52}$ S. Park, B. Lee, S. H. Jeon, and S. Han, Curr. Appl. Phys. 11, S337 - S340 (2011).

${ }^{53}$ L. Y. Lim, S. Lany, Y. J. Chang, E. Rotenberg, A. Zunger, and M. F. Toney, Phys. Rev. B 86, 235113 (2012)

${ }^{54}$ B. Himmetoglu, A. Floris, S. de Gironcoli, and M. Cococcioni, Int. J. Quantum Chem. 114, 14-49 (2014)

${ }^{55}$ J. Paier, M. Marsman, K. Hummer, G. Kresse, I. C. Gerber, and J. G. Ángyán, J. Chem. Phys. 124, 154709 (2006)

${ }^{56}$ F. Fuchs, J. Furthmüller, F. Bechstedt, M. Shishkin, and G. Kresse, Phys. Rev. B 76, 115109 (2007)

${ }^{57}$ V. Ivády, I. A. Abrikosov, E. Janzén, and A. Gali, Phys. Rev. B 87, 205201 (2013)

${ }^{58}$ R. C. Albers, N. E. Christensen, and A. Svane, J. Phys.: Condens. Matter 21, 343201 (2009).

${ }^{59}$ F. Oba, M. Choi, A. Togo, and I. Tanaka, Sci. Technol. Adv. Mater. 12, 034302 (2011)

${ }^{60} \mathrm{~W}$. Martienssen and H. Warlimont, eds., (Springer Berlin, Heilderberg, 2005).

${ }^{61}$ S. Kasap and P. Capper, eds., (Springer-Verlag New York, Inc., Secaucus, NJ, USA, 2007).

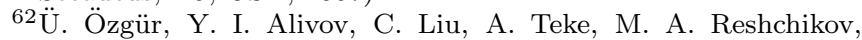
S. Doğan, V. Avrutin, S.-J. Cho, and H. Morkoç, J. Appl. Phys. 98, 041301 (2005).

${ }^{63}$ L. Ley, R. A. Pollak, F. R. McFeely, S. P. Kowalczyk, and D. A. Shirley, Phys. Rev. B 9, 600-621 (1974)

${ }^{64}$ Y. Dou, R. G. Egdell, D. S. L. Law, N. M. Harrison, and B. G. Searle, J. Phys.: Condens. Matter 10, 8447 (1998).

${ }^{65}$ A. Seidl, A. Görling, P. Vogl, J. A. Majewski, and M. Levy, Phys. Rev. B 53, 3764-3774 (1996)

${ }^{66}$ V. I. Anisimov, I. V. Solovyev, M. A. Korotin, M. T. Czyzyk, and G. A. Sawatzky, Phys. Rev. B 48, 16929-16934 (1993)

${ }^{67}$ A. V. Krukau, O. A. Vydrov, A. F. Izmaylov, and G. E. Scuseria, J. Chem. Phys. 125, 224106 (2006)

${ }^{68}$ P. E. Blöchl, Phys. Rev. B 50, 17953-17979 (1994).

${ }^{69}$ G. Kresse and J. Furthmüller, Comput. Mater. Sci. 6, 15-50 (1996).

${ }^{70}$ G. Kresse and D. Joubert, Phys. Rev. B 59, 1758-1775 (1999).

${ }^{71}$ H. J. Monkhorst and J. D. Pack, Phys. Rev. B 13, 5188-5192 (1976).

${ }^{72}$ E. N. Brothers, A. F. Izmaylov, J. O. Normand, V. Barone, and G. E. Scuseria, The Journal of Chemical Physics 129, 011102 (2008).

${ }^{75}$ B. G. Janesko, T. M. Henderson, and G. E. Scuseria, Phys. Chem. Chem. Phys. 11, 443-454 (2009)

${ }^{{ }^{4}}$ M. Shishkin and G. Kresse, Phys. Rev. B 75, 235102 (2007)

${ }^{75}$ See supplemental material (Figs. S1-S12) at [URL to be inserted by AIP] for the total and projected density of states of the II-VI semiconductors under consideration.

${ }^{76}$ W. A. Harrison, Phys. Rev. B 31, 2121-2132 (1985)

${ }^{77}$ J. Paier, M. Marsman, and G. Kresse, Phys. Rev. B 78, 121201 (2008)

${ }^{8}$ See supplemental material (Fig. S13) at [URL to be inserted by AIP] for a plot of $\Delta \varepsilon_{d}^{*}$ versus $U^{*}$.

${ }^{79}$ S. Adachi, ed., Vol. 3: II-VI Compound Semiconductors (Kluwer Academic Publishers, Boston, MA, 2004).

${ }^{80} \mathrm{H}$. Finkenrath and N. Uhle, Solid State Commun. 5, $875-877$ (1967)

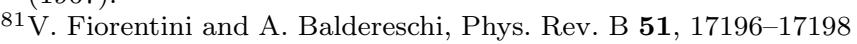
(1995)

${ }^{82}$ W. von der Linden, P. Horsch, and W.-D. Lukas, Solid State Commun. 59, $485-490$ (1986)

\section{SUPPLEMENTAL MATERIAL}

The total (DOS) and projected (PDOS) density of states of zinc and cadmium monochalcogenides, obtained via density-functional (PBE), hybrid-functional (HSE), and combined $\mathrm{HSE}+U^{*}$ calculations, are given Figs. S1S12. Figure S13 shows a plot of the difference $\Delta \varepsilon_{d}^{*}=$ $\varepsilon_{d}^{\mathrm{HSE}+U^{*}}-\varepsilon_{d}^{\mathrm{HSE}}$ versus the optimal Hubbard parameter $U^{*}$. 
$w-\mathrm{ZnO}$
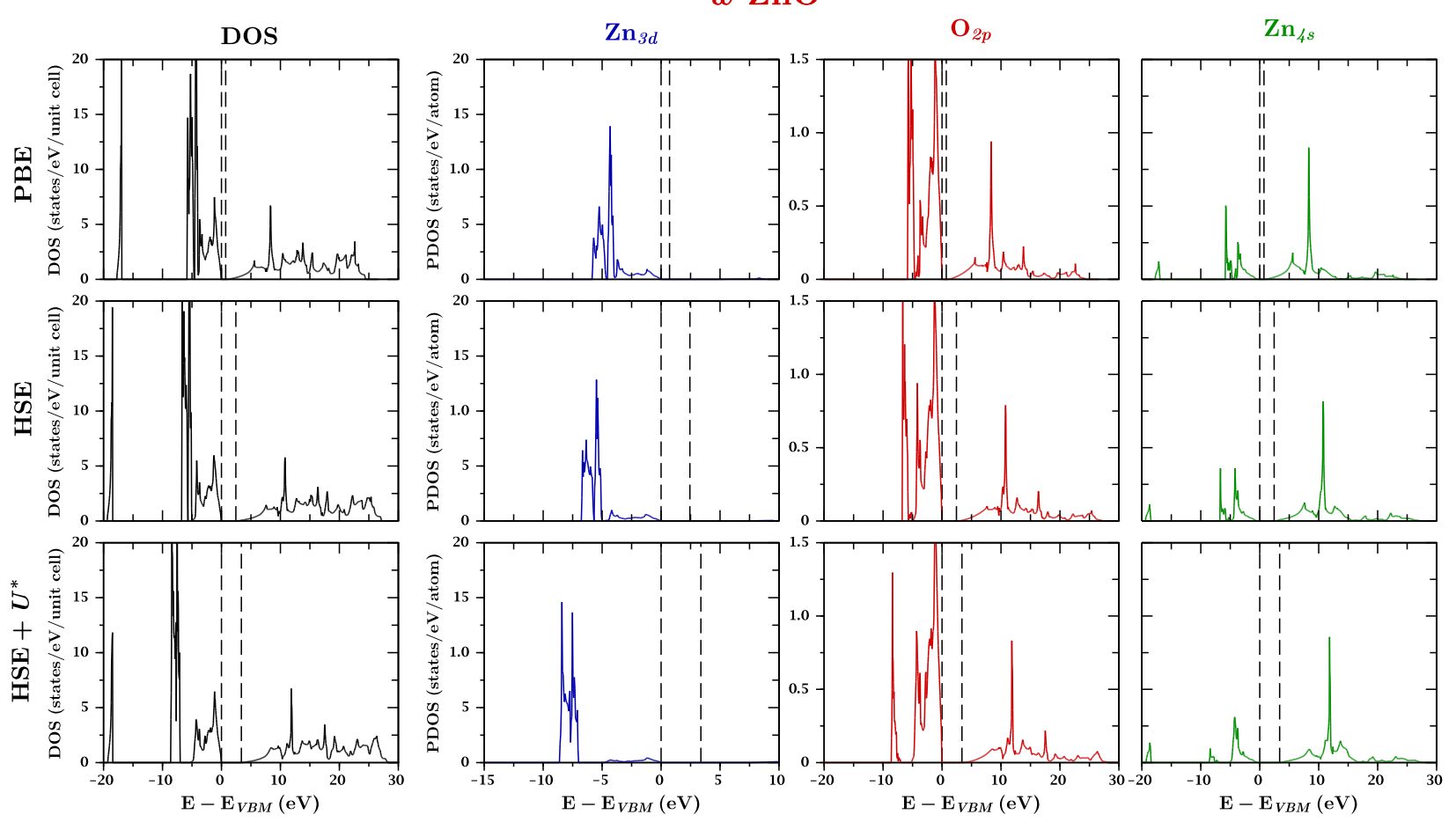

FIG. S7. The total and projected density of states of $w$-ZnO.
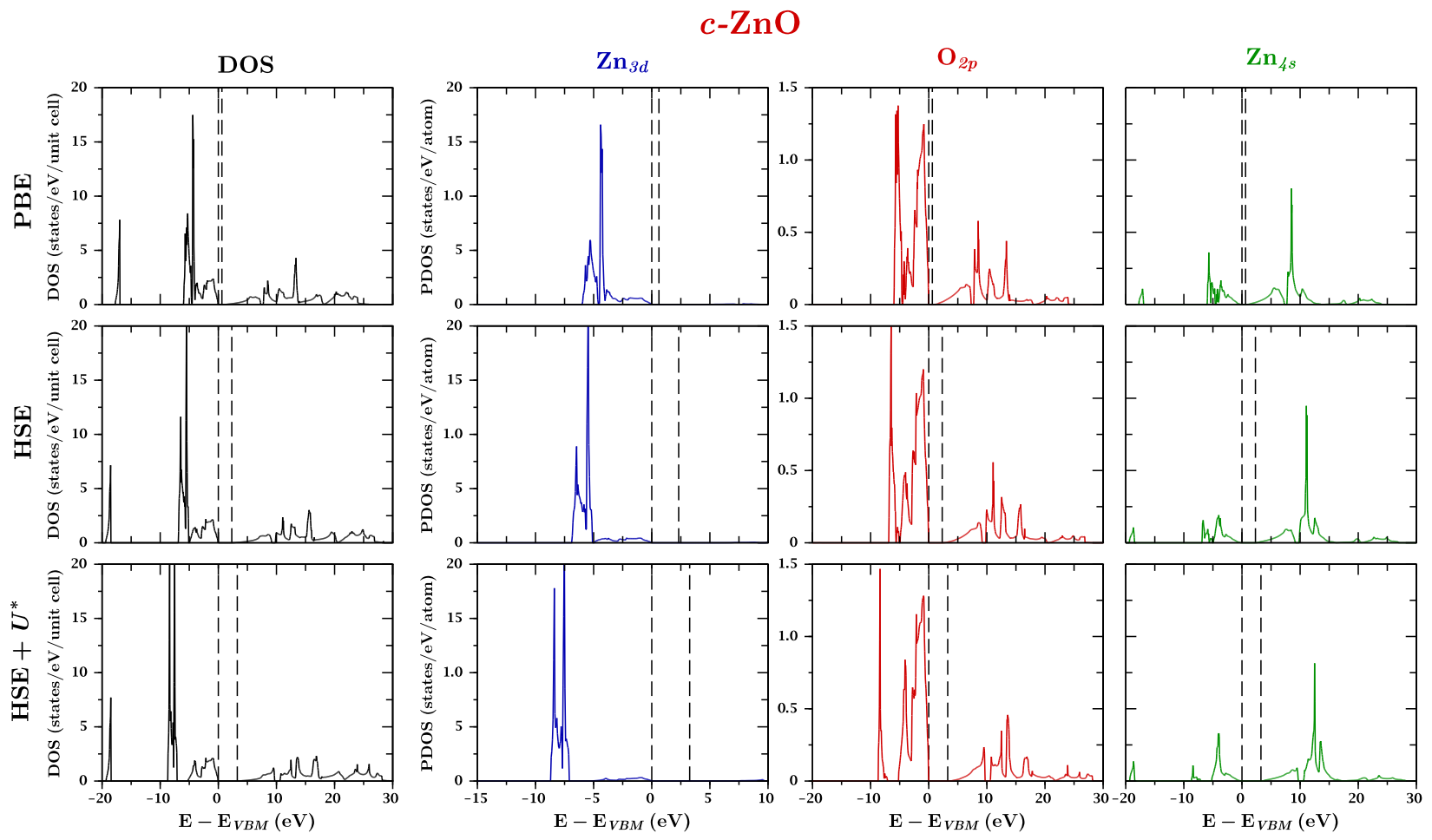

FIG. S8. The total and projected density of states of $c-\mathrm{ZnO}$. 


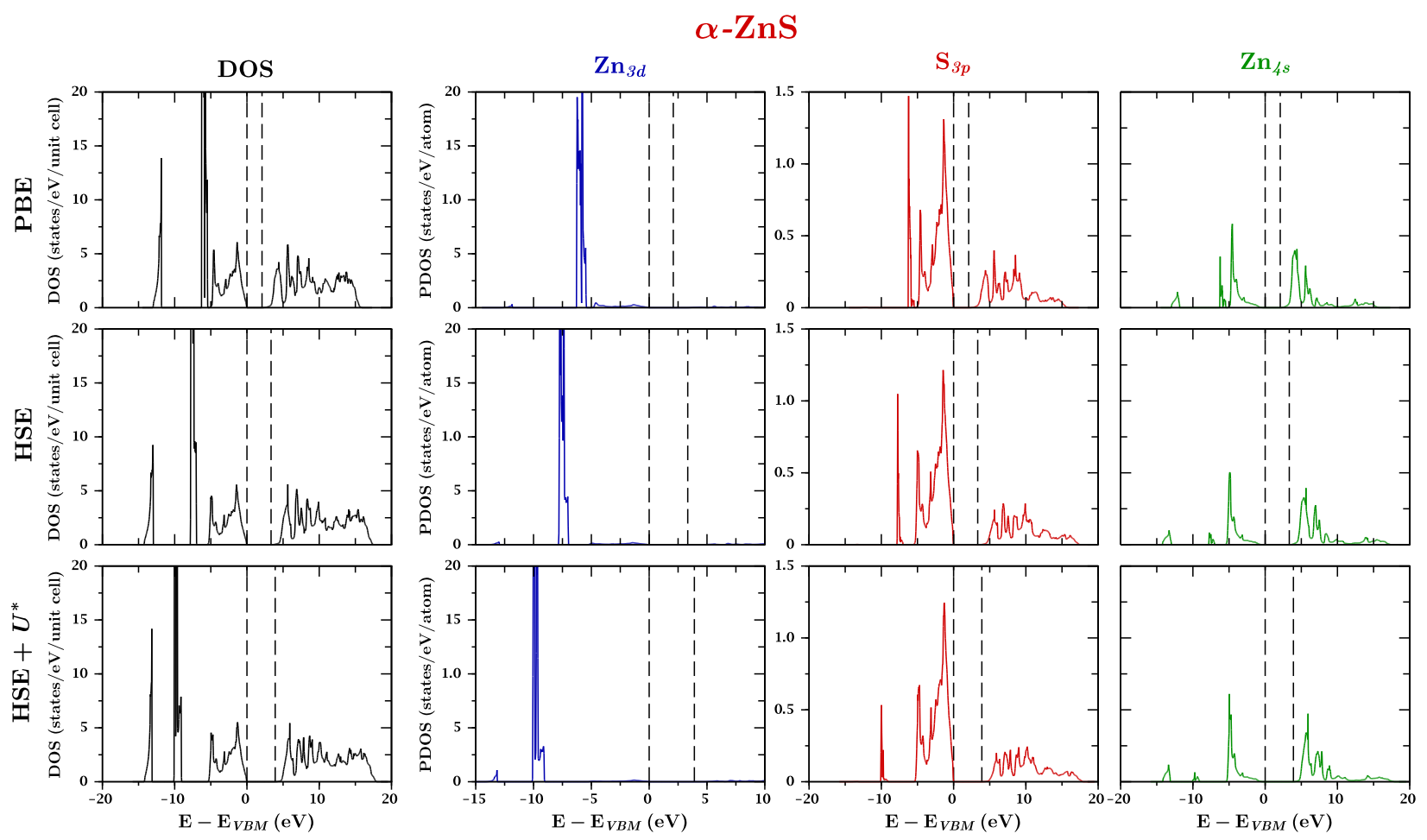

FIG. S9. The total and projected density of states of $\alpha$-ZnS.
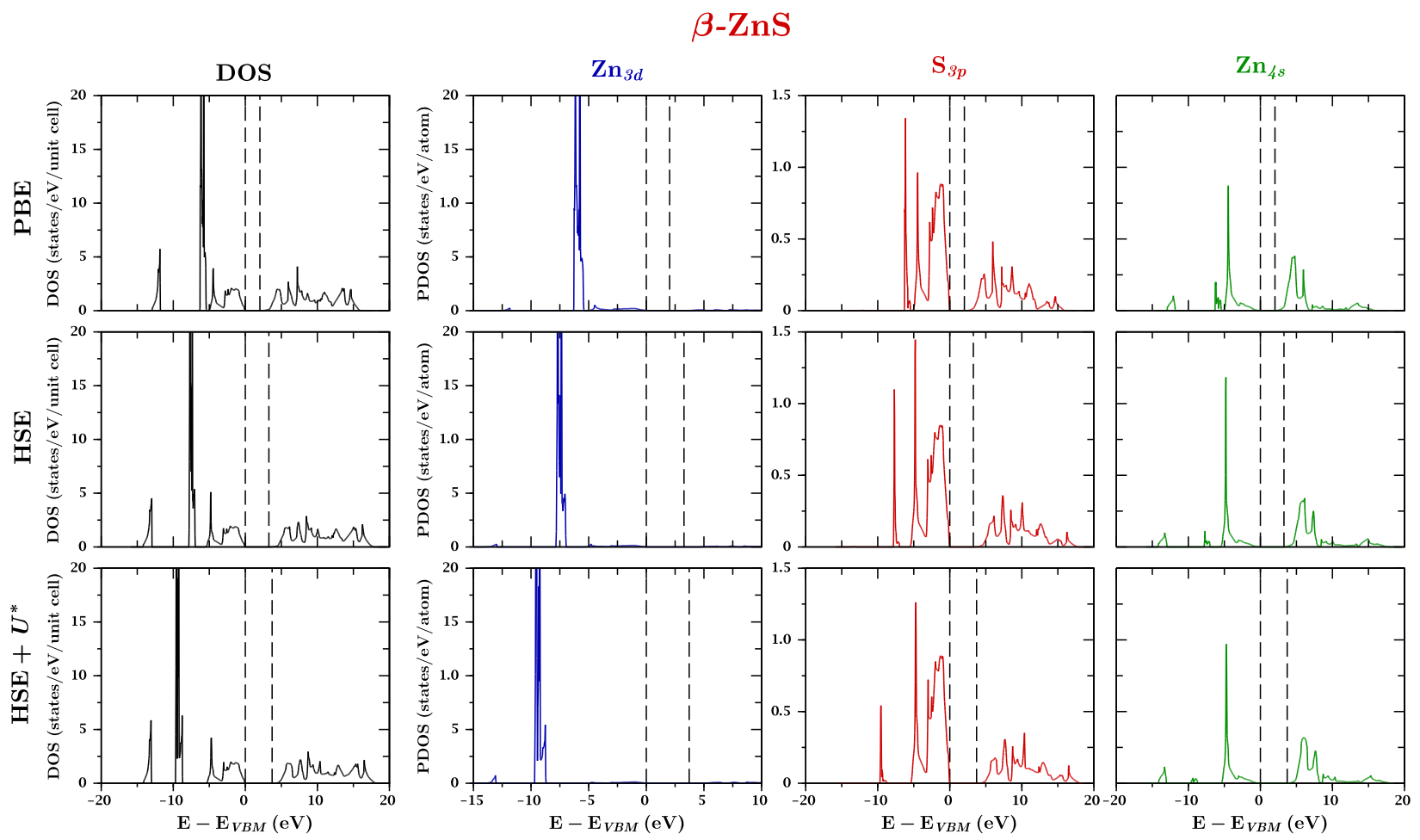

FIG. S10. The total and projected density of states of $\beta-\mathrm{ZnS}$. 
ZnSe
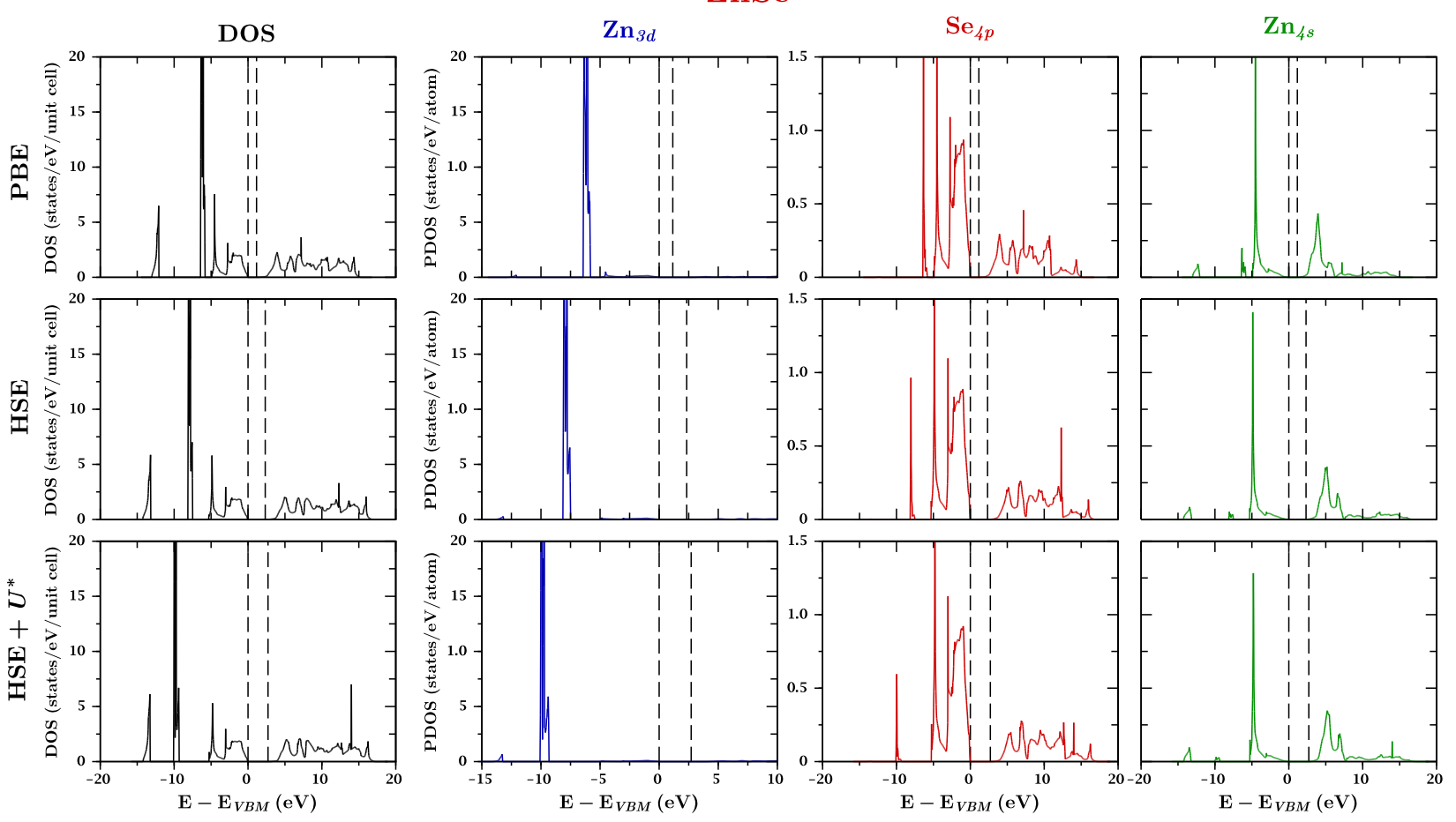

FIG. S11. The total and projected density of states of ZnSe.
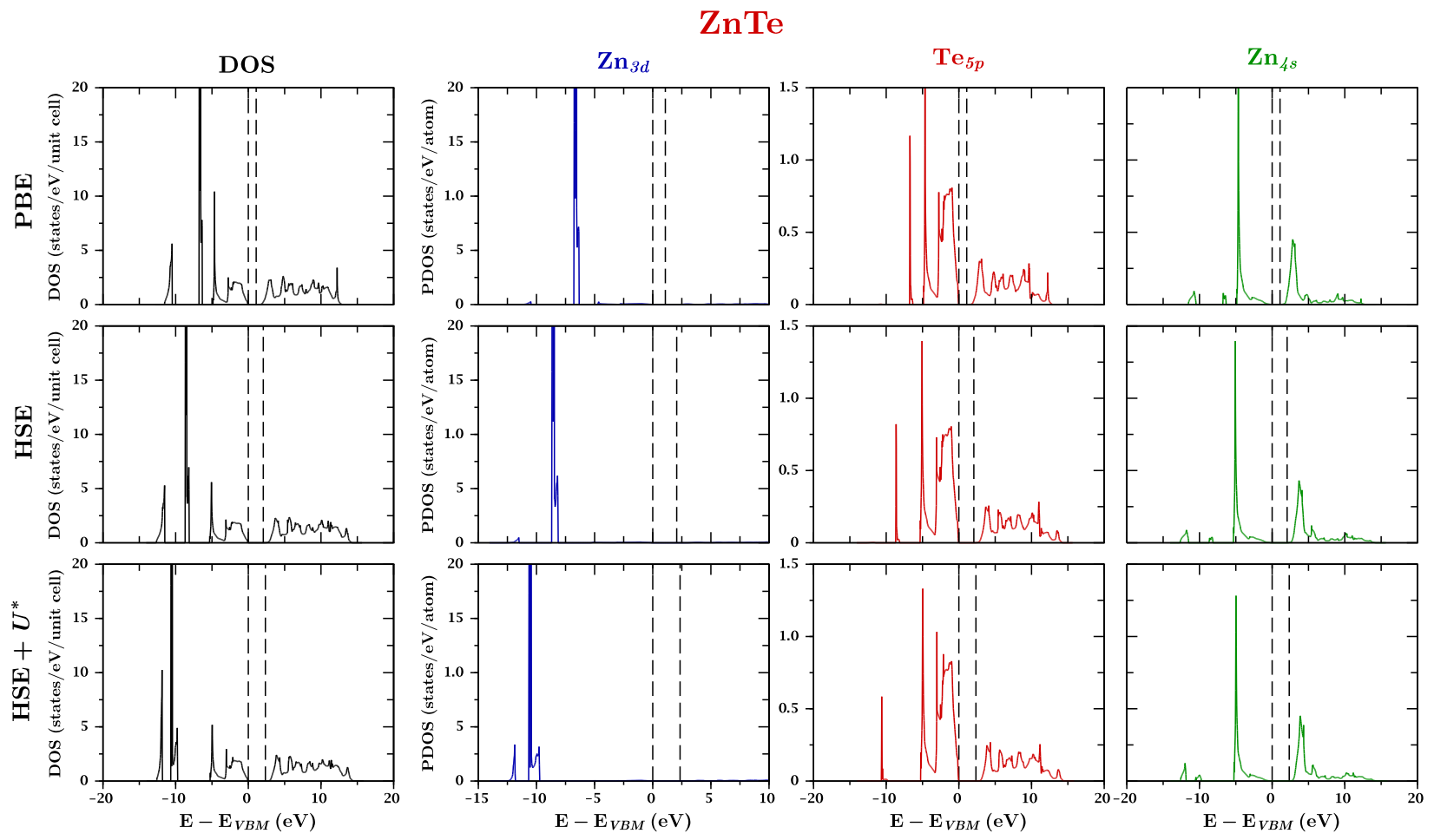

FIG. S12. The total and projected density of states of ZnTe. 

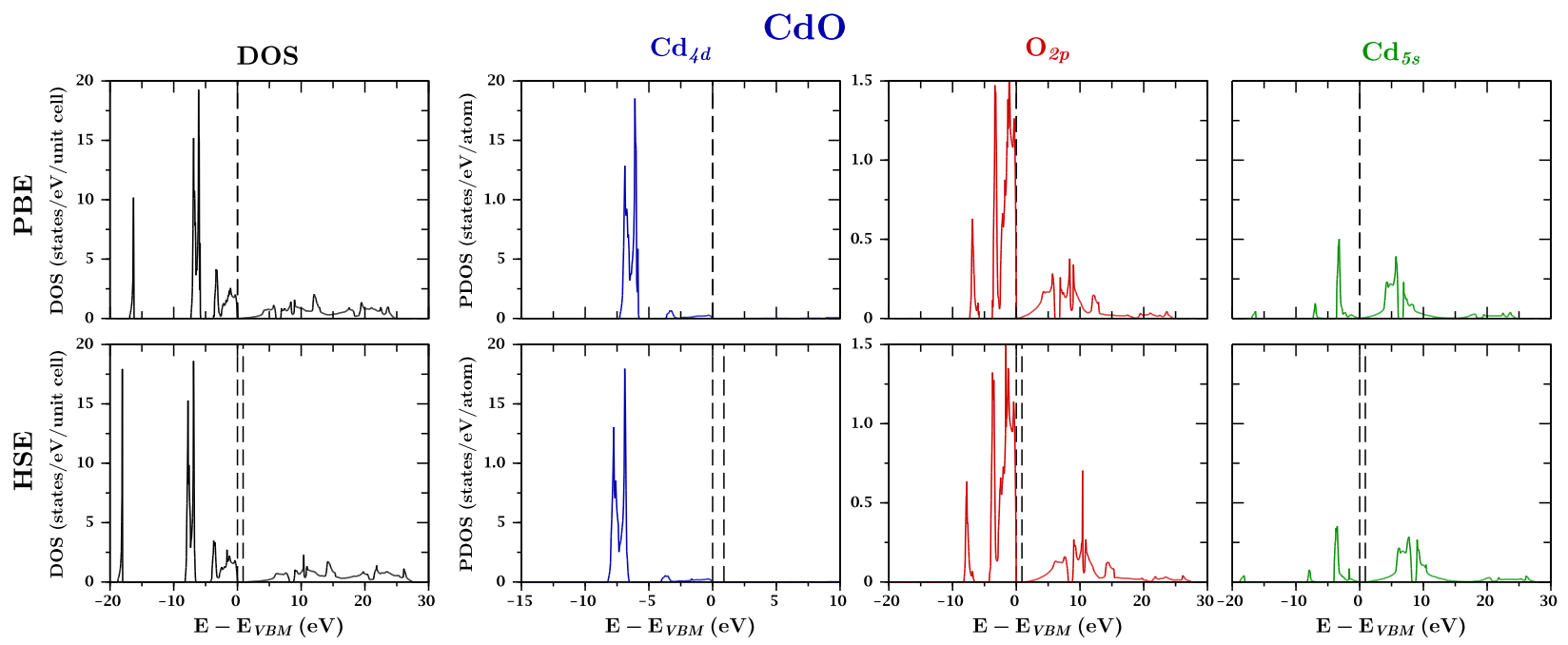

FIG. S13. The total and projected density of states of CdO.
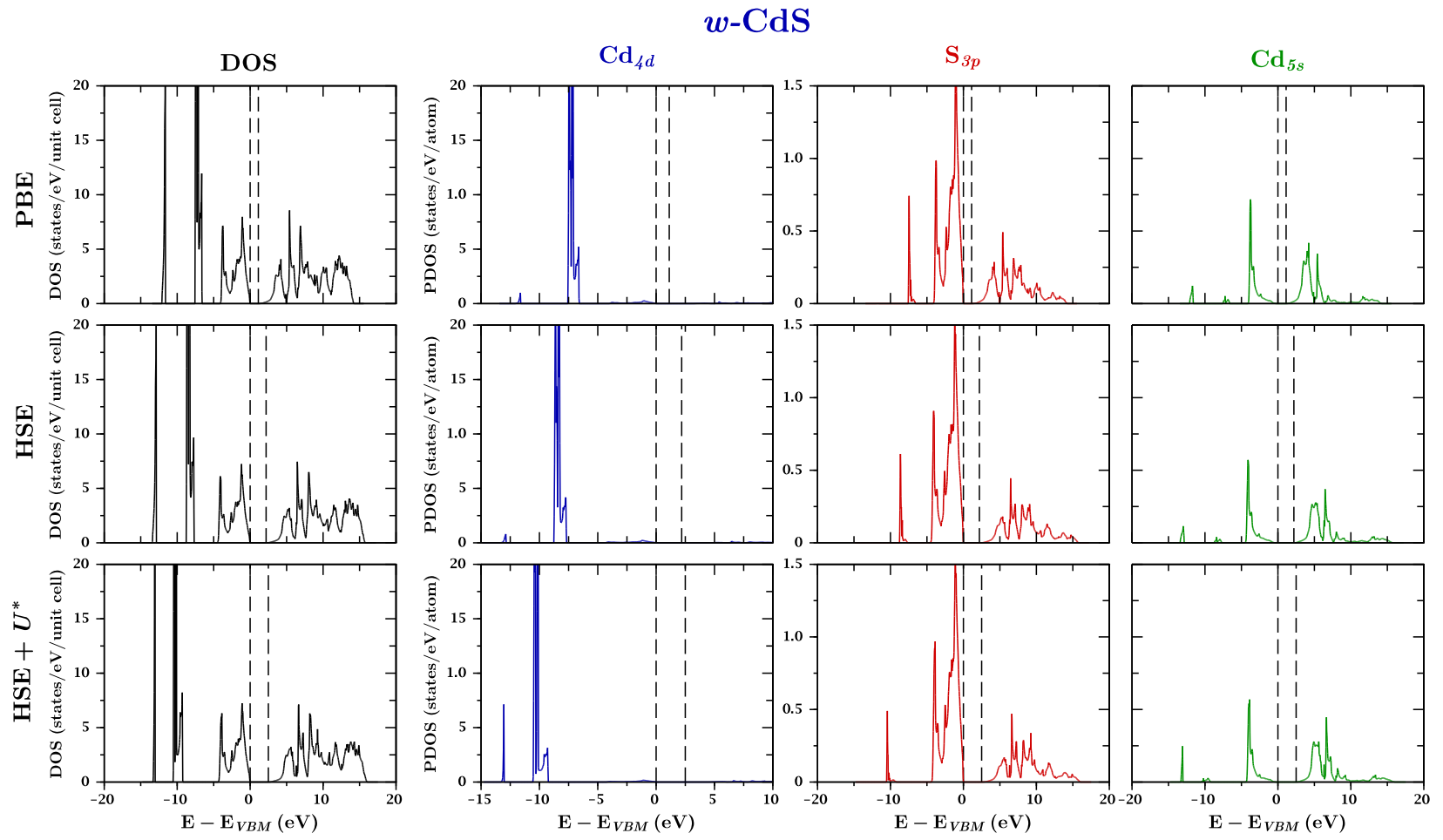

FIG. S14. The total and projected density of states of $w$-CdS. 


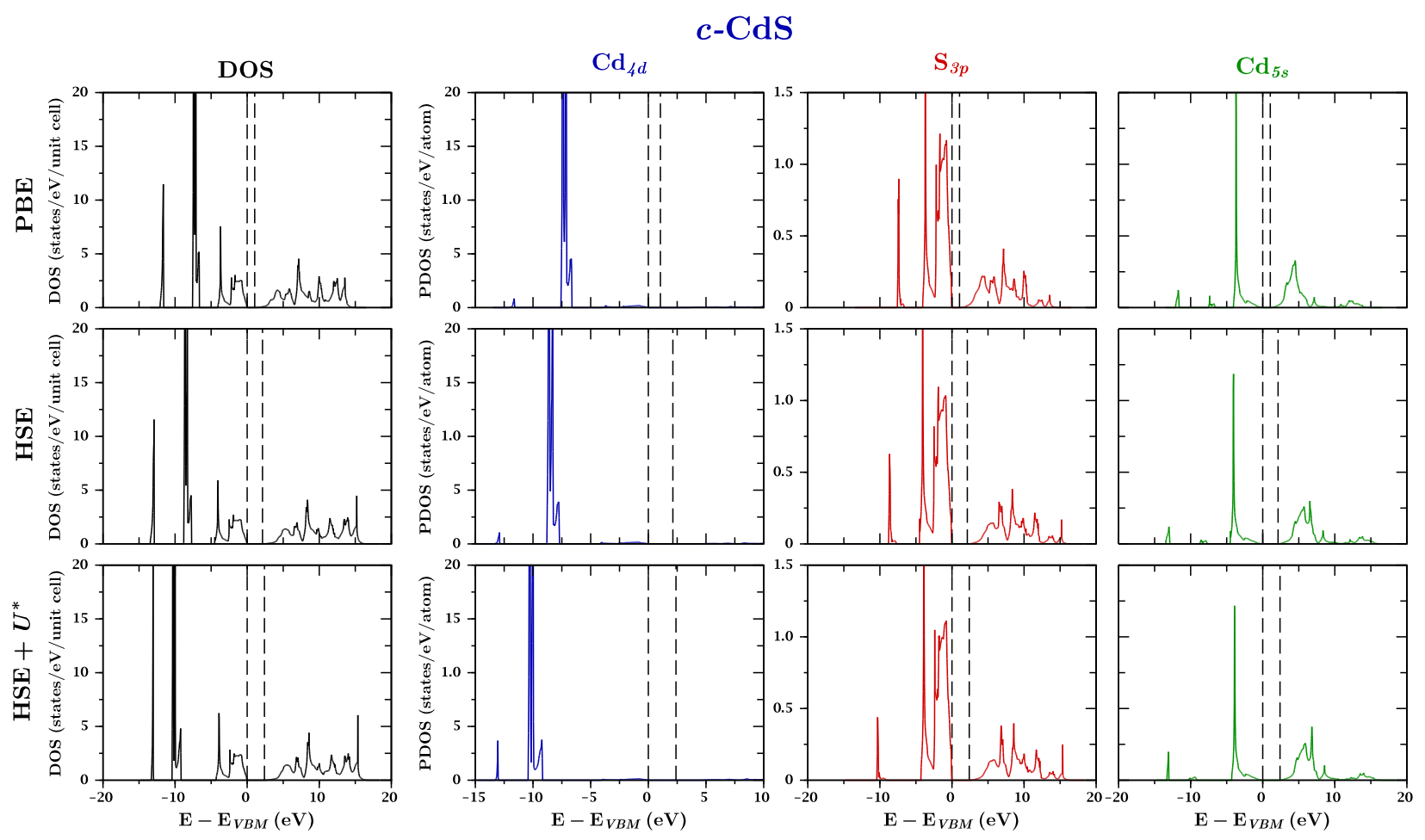

FIG. S15. The total and projected density of states of $c$-CdS.
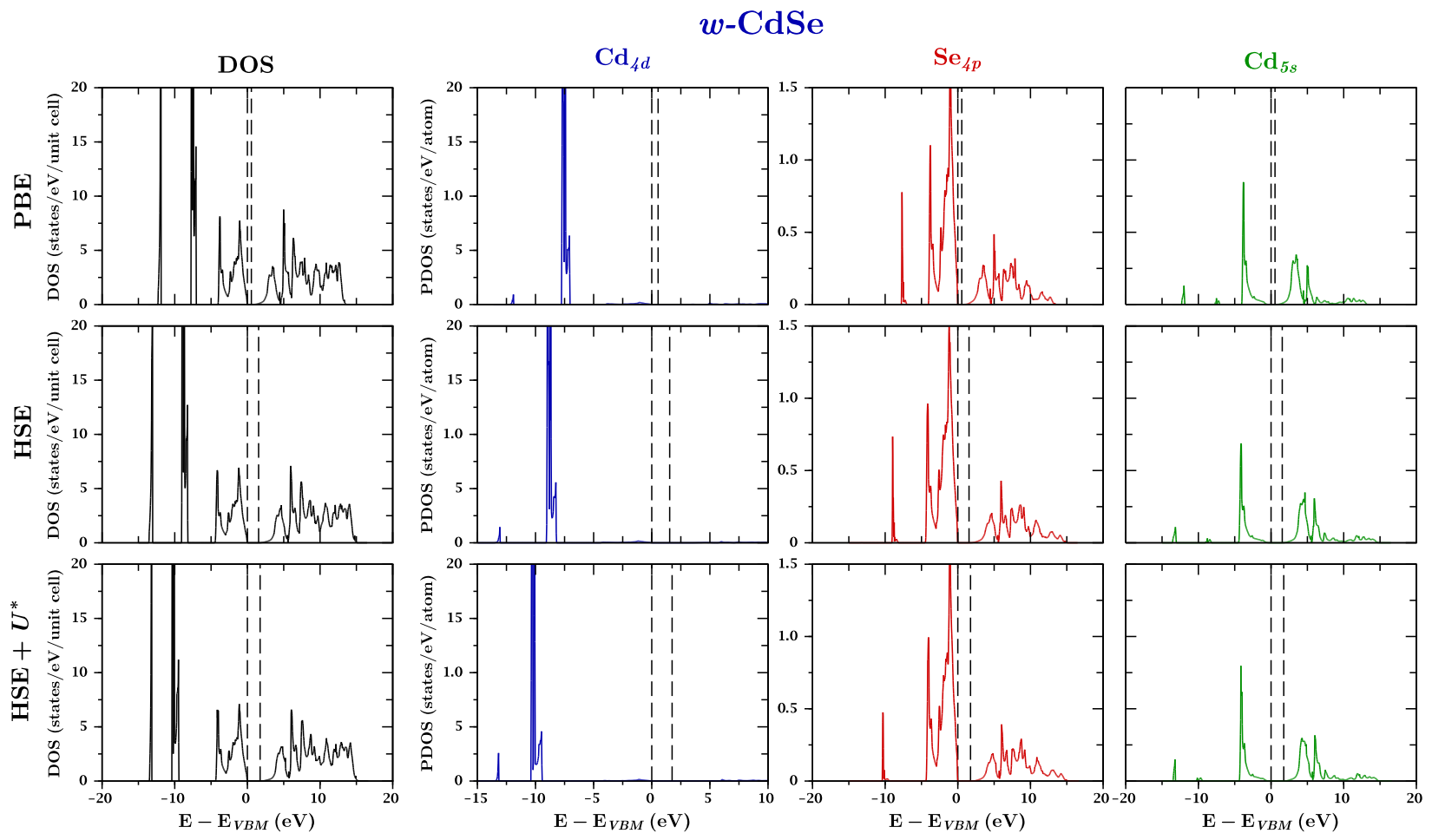

FIG. S16. The total and projected density of states of $w$-CdSe. 


\section{$c-\mathrm{CdSe}$}
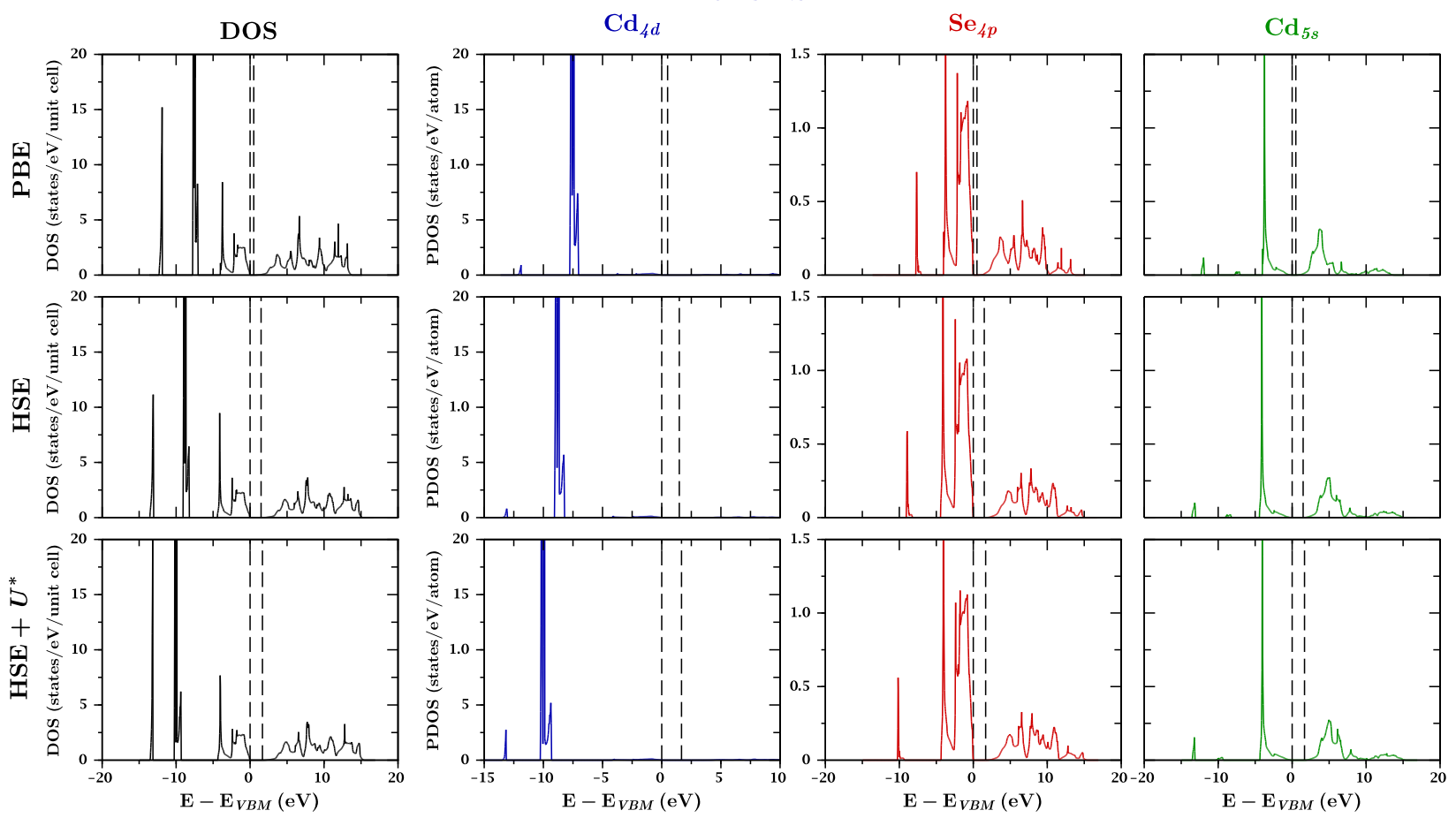

FIG. S17. The total and projected density of states of $c$-CdSe.
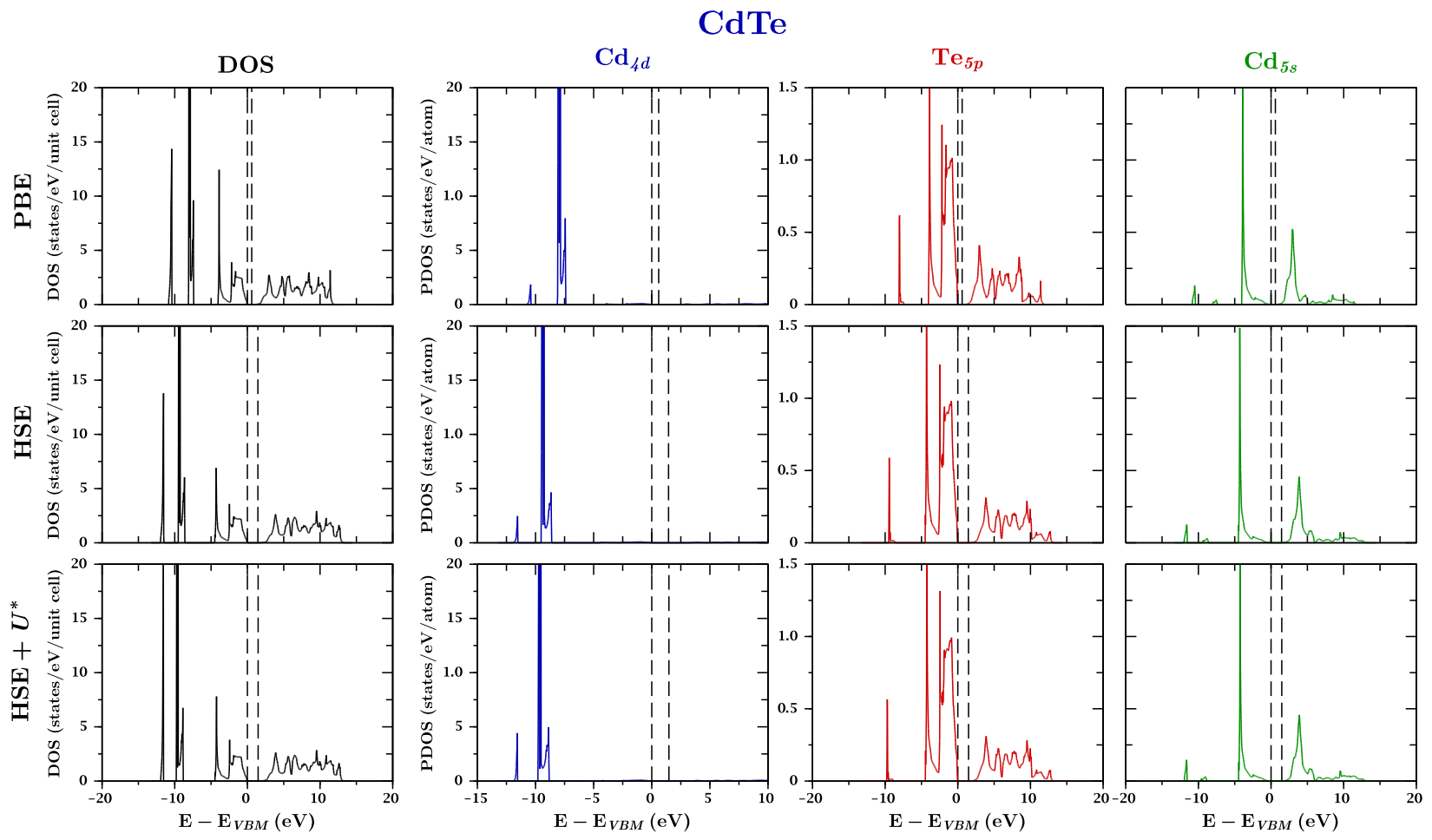

FIG. S18. The total and projected density of states of CdTe. 


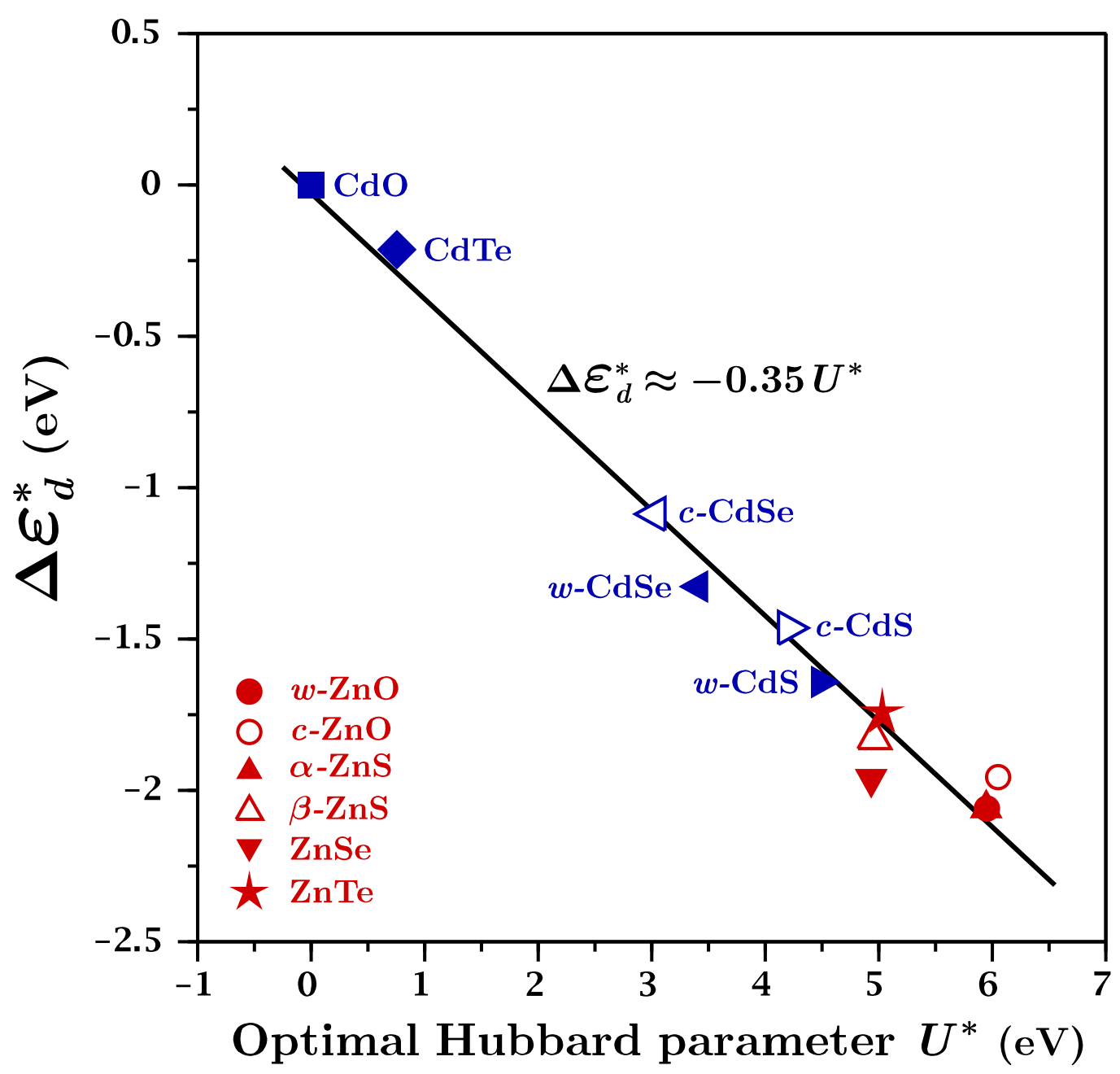

FIG. S19. (Color online) The difference $\Delta \varepsilon_{d}^{*}=\varepsilon_{d}^{\mathrm{HSE}+U^{*}}-$ $\varepsilon_{d}^{\mathrm{HSE}}$ as a function of the optimal Hubbard parameter $U^{*}$. 